\title{
EVALUATING THE UPTAKE OF TEN HEAVY METALS BY KIDNEY BEAN (PHASEOLUS VULGARIS L.) GROWN IN A SOIL- SLUDGE MIXTURE USING A REGRESSION MODEL
}

\author{
Eid, E. M..$^{1,2^{*}}$ - Shaltout, K. H. ${ }^{3}$ - AlAmRI, S. A. M. ${ }^{1,4}$ - SEWElAM, N. A. ${ }^{3}$ - GAlAL, T. M. ${ }^{5}$ \\ ${ }^{1}$ Biology Department, College of Science, King Khalid University, Abha 61321, P. O. Box 9004, \\ Saudi Arabia
}

${ }^{2}$ Botany Department, Faculty of Science, Kafr El-Sheikh University, Kafr El-Sheikh 33516, Egypt

${ }^{3}$ Botany Department, Faculty of Science, Tanta University, Tanta 31527, Egypt

${ }^{4}$ Prince Sultan Bin Abdul-Aziz Center for Environment and Tourism Research and Studies, King Khalid University, Abha 61421, P. O. Box 960, Saudi Arabia

${ }^{5}$ Botany and Microbiology Department, Faculty of Science, Helwan University, Cairo, Egypt

*Corresponding author

e-mail:eeid@kku.edu.sa,ebrahem.eid@sci.kfs.edu.eg,ebrahem.eid@gmail.com; phone: +96655-271-7026; fax: +966-17-241-8205

(Received 20 $0^{\text {th }}$ Feb 2020; accepted 25 $5^{\text {th }}$ May 2020)

\begin{abstract}
Severe human health risks can be caused by consuming vegetables contaminated by heavy metals (HMs); thus, assessing the HM uptake by these plants is important. The current work was performed to construct a regression model for predicting the concentration of ten HMs in four tissues of Phaseolus vulgaris (roots, stems, leaves and pods) based on their concentration in a soil-sludge mixture, soil organic matter (OM) and soil $\mathrm{pH}$. For pods, the regression equation with the highest coefficient of determination $\left(R^{2}=0.99\right)$ and model efficiency $(M E=1.00)$ but the lowest mean normalized bias $(M N B=0.01)$ was that of cobalt. For leaves, the equation with the highest $R^{2}(0.90)$ and $M E(0.92)$ but the lowest $M N B(0.001)$ was that of molybdenum. Comparable findings were obtained for molybdenum in the stems and manganese in the roots. All $t$ values that assessed the difference between the actual and predicted values of the ten HMs in the four tissues were nonsignificant. Thus, these models could be used as a risk assessment tool for $P$. vulgaris cultivated in soil-sludge combinations.
\end{abstract}

Keywords: bioconcentration factor, prediction modelling, sewage sludge, translocation factor, vegetable crops

\section{Introduction}

Contamination of cultivated lands by heavy metals (HMs) due to severe human impacts (such as industrial effluents, sewage sludge, chemical fertilizers and pesticides, untreated irrigation water contaminated by wastewater and mining activities) represents a hazardous global problem (Ramadan and Al-Ashkar, 2007). Some HMs are essential to plants at low concentrations (e.g., $\mathrm{Zn}, \mathrm{Mn}, \mathrm{Fe}$ and $\mathrm{Cu}$ ), but others pose a toxicity risk to living organisms (e.g., $\mathrm{Ni}, \mathrm{Cd}, \mathrm{Pb}, \mathrm{As}, \mathrm{Hg}$ and $\mathrm{Cr}$ ). Therefore, many studies have evaluated the risks associated with the accumulation of HMs in soil-crop systems (Belaid et al., 2012; Galal, 2016; Farahat et al., 2017; Jamali et al., 2007, 2009; Zhao et al., 2010).

Although HMs occur in different forms in the soil, only the dissolved forms are available for uptake by plants (Aydinalp and Marinova, 2003). The adsorption and desorption properties of soil are among the main factors that limit the bioavailability of HMs for plants (Krishnamurti et al., 1999). Some soil factors, such as $\mathrm{pH}$, organic matter (OM), and Fe and Mn oxides, influence the adsorption and desorption of HMs in 
soil (Usman et al., 2008). In view of this, soil $\mathrm{pH}$ has a significant negative correlation with the mobility and availability of HMs in soil solution, with acidity increasing the availability of HMs and alkalinity having a decreasing effect (Du Laing et al., 2008; Novotná et al., 2015; Zeng et al., 2011).

Consequent to the treatment of drainage water resulting from municipal and industrial activities, sewage sludge (SS) is produced as an organic byproduct that has excess macro- and micro-nutrients (Eid and Shaltout, 2016). Approximately 1.3 billion tons of SS is produced by cities globally per annum, and this is predicted to expand to 2.2 billion tons globally per annum by 2050 worldwide (Hoornweg and Bhada-Tata, 2012). Thus, there is consideration for how such quantities of SS can be disposed of safely to ensure it does not impact the environment (Eid et al., 2017) with the best means indicated as landfills and land application (Li et al., 2018). Some studies have indicated that the mixing of cultivated soil with certain amounts of SS has an enhancing effect on soil fertility and plant nutrients (Aráujo et al., 2007). Many studies (Singh and Agrawal, 2008, 2010; Grotto et al., 2015; Rehman et al., 2018; Asgari Lajayer et al., 2019) reported that SS could be added as a valuable fertilizer that improves the growth and yield of plant crops. However, this practice may lead to a risk to humans and the environment because of the presence of HMs that could contaminate soil and water, cause phytotoxicity, and the entry of such contaminants into the food supply (Mamais et al., 2000; Dolgen et al., 2007; Singh and Agrawal, 2007). The degree of danger is dependent on the quantity of SS applied, its structure, the crop species used and the conventional controls employed (Latare et al., 2014).

To reduce the toxicity levels of HMs and their movement through the food chain, it is important to assess the impact of soil variables on the availability and uptake of HMs by plants (Zeng et al., 2011). Regression models are useful mathematical tools for predicting the concentration of HMs in crop plants using certain soil factors such as HMs, pH and OM (Waegeneers et al., 2011). Comparable equations were constructed for barley (Adams et al., 2004; Novotná et al., 2015; Eid et al., 2020a), broad bean (Eid et al., 2019), carrot (Legind and Trapp, 2010; Bešter et al., 2013), cauliflower (Kumar et al., 2019), celery (Wang et al., 2004), chicory (Bešter et al., 2013), Chinese cabbage (Zhang et al., 2016), cucumber (Eid et al., 2018a), endive (Bešter et al., 2013), Eruca sativa (Eid et al., 2020b), garden pea (Eid et al., 2020c), hop (Novotná et al., 2015), lettuce (Legind and Trapp, 2010), maize (Tudoreanu and Phillips, 2004; Yang et al., 2013; Novotná et al., 2015), onion (Bešter et al., 2013), potato (Bešter et al., 2013; Novotná et al., 2015), rapeseeds (Novotná et al., 2015), red beet (Bešter et al., 2013), rice (Zeng et al., 2011; Rafiq et al., 2014), rye grass (Tudoreanu and Phillips, 2004), soy bean (Tudoreanu and Phillips, 2004), spinach (Wang et al., 2004; Eid et al., 2018b), tomato (Ramadan and Al-Ashkar, 2007; Bešter et al., 2013), wheat (Adams et al., 2004; Chaudri et al., 2007; Novotná et al., 2015; Eid et al., 2020d) and zucchini (Bešter et al., 2013). However, differences in soil, climate, SS composition and management factors require more specific estimates for different climatic regions or different cropping systems (Binder et al., 2002). Therefore, for safe crop production, it is important to evaluate the toxic levels of the HMs in the soil by establishing regression equations between the HMs in the plants on the one hand and those in the soil supporting the plants on the other hand (Eid et al., 2020d).

The kidney bean (Phaseolus vulgaris L.) is a herbaceous annual leguminous plant that has a long history of cultivation (Gentry, 1969). P. vulgaris is an annual plant belonging to family Fabaceae that originated in Central-South America. It is an erect or 
twining plant with compound leaves (each with three leaflets), and its flowers are usually white to purple in colour. Its pods are long, narrow, and straight or slightly curved, with pointed tips. The size and colour of its seeds are variable. The pods are cooked in immature stages, leaves are edible, and dried seeds are cooked and sometimes fried in oils as a salad (Huxley, 1999; Tindall, 1978). P. vulgaris is the most important food legume for direct consumption in the world (Jones, 1999). It was therefore selected for assessment in this research study. To the best of the authors' knowledge, no regression models are available for the uptake of HMs by $P$. vulgaris grown in soil amended with SS. Therefore, the present study was carried out to develop a mathematical model for predicting the potential uptake of HMs by $P$. vulgaris after growing it in an agricultural soil with different rates of SS addition under greenhouse conditions. Such models are suitable for assessing the severe impact of cultivating some vegetable crops in soil mixed with different amounts of SS.

\section{Materials and methods}

\section{Materials and experimental design}

White seeds of P. vulgaris (Strike, Seminis Vegetable Seeds, Inc., St. Louis, USA) were obtained from a local market in Abha City, Saudi Arabia. The soil used in the experiment was collected from nearby cultivated fields at a depth of $0-20 \mathrm{~cm}$ (Lat.: $18^{\circ}$ $14^{\prime} 36.37^{\prime \prime} \mathrm{N}$, Long.: $42^{\circ} 33^{\prime} 58.25^{\prime \prime} \mathrm{E}$ ), while the SS was obtained from the municipal sewage treatment station in Abha (Lat.: $18^{\circ} 13^{\prime} 59.19^{\prime \prime} \mathrm{N}$, Long.: $42^{\circ} 31^{\prime} 16.35^{\prime \prime} \mathrm{E}$ ). This station treats some $41,275 \mathrm{~m}^{3}$ day $^{-1}$ wastewater and produces 90 tons day ${ }^{-1}$ dry SS (Eid et al., 2017). Soil and SS samples were air dried for 2 weeks, ground and passed through a 2-mm sieve.

The experiment was performed in the greenhouse of the Biology Department, King Khalid University, Abha. Based on a preliminary experiment, the SS was mixed with agricultural soil at concentrations of $0,10,20,30,40$ and $50 \mathrm{~g} \mathrm{~kg}^{-1}$. Each treatment consisted of six plastic pots (each with a 6-L volume, filled with four $\mathrm{kg}$ of the respective treatment, and planted with ten equally sized seeds). The experimental units were arranged following a completely randomized design (CRD). The seeds were sown (directly after mixing the soil with SS) on the $4^{\text {th }}$ of January 2018 for a period of 57 days under a natural day/night regime, irrigated using tap water as required with the soil water holding capacity (SWHC) in each pot varying between $40-50 \%$ and weeded manually as needed. The SWHC was determined and maintained on a volume basis using the following formula (Eq. 1):

$$
\text { Volumetric SWHC }(\%)=(\text { depth of water in } \mathrm{cm} / \text { depth of soil in } \mathrm{cm}) \times 100
$$

Fifteen days after sowing, the emerged plants were manually thinned to one individual in each pot.

\section{Sample analysis}

Plant materials were harvested on 1 March 2018, then cleaned under running tap water, separated into four tissues (roots, pods, stems and leaves), dried for one week at $60{ }^{\circ} \mathrm{C}$, powdered in a plastic mill and stored for later use. For physicochemical analyses, post-harvest soil samples were air-dried for two weeks, ground and passed through a 2- 
mm sieve. Dried powders (pre- and post-harvest soil and SS samples) were analysed for OM using the loss-on-ignition method at $550{ }^{\circ} \mathrm{C}$ for $2 \mathrm{~h}$ (Wilke, 2005), while $\mathrm{pH}$ was measured in 1:5 soil:water extracts (Allen, 1989).

For HM estimation, 0.5-1.0 g of each dried powders and plant sample was digested following the mixed-acid digestion method $\left(\mathrm{HNO}_{3}\right.$ and $\mathrm{HClO}_{4} ; 3: 1$, v/v). A microwave sample preparation system was used for digestion (PerkinElmer Titan MPS, PerkinElmer Inc., USA). Blank samples were used to verify the accuracy of the digestion procedure and the subsequent analyses. Ten $\mathrm{HMs}(\mathrm{Cd}, \mathrm{Co}, \mathrm{Cr}, \mathrm{Cu}, \mathrm{Fe}, \mathrm{Mn}$, $\mathrm{Mo}, \mathrm{Ni}, \mathrm{Pb}$ and $\mathrm{Zn}$ ) were estimated by inductively coupled plasma optical emission spectrometry (Thermo Scientific iCAP 7000 Plus Series; Thermo Fisher Scientific, USA) as outlined by Allen (1989). The detection limits of the HMs (in $\mu \mathrm{g}^{-1}$ ) were as follows: 6.0 for $\mathrm{Ni} ; 2.0$ for $\mathrm{Co}, \mathrm{Cr}$ and $\mathrm{Cu} ; 1.0$ for $\mathrm{Fe}, \mathrm{Pb}$ and $\mathrm{Zn}$; 0.3 for $\mathrm{Mn}$; and 0.1 for $\mathrm{Cd}$ and Mo. The settings of the instrument and the conditions of operation were as specified by the manufacturer. Standard solutions with known concentrations of the ten HMs were prepared for system standardization.

\section{Quality assurance and control}

Certified reference material (tomato leaves, SRM 1573a) was used to verify the accuracy of HM estimates. The reference material was digested and analysed using the methods applied to the $P$. vulgaris samples. Digestion and measurement of the ten HMs were performed in triplicate. Accuracy was assessed by comparing the actual concentration with the certified value and then expressed as a percentage. Recovery rates had a range of $95-104 \%$ for SRM 1573a.

\section{Data analysis}

Before performing the one-way analysis of variance (ANOVA-1), the distribution of the HM, bioconcentration factor (BCF) and translocation factor (TF) data was tested for normality using the Shapiro-Wilk $W$ test and for homogeneity of variance using Levene's test; when necessary, the data were log-transformed. The HM data for $P$. vulgaris tissues were subjected to an ANOVA-1 to evaluate the differences among $P$. vulgaris tissues. Significant difference between means among the four tissues were identified using the Tukey's HSD test at $\mathrm{p}<0.05$. The BCF determines the capacity of a plant, such as P. vulgaris, to accumulate an HM in its roots (Galal et al., 2017) and is calculated using the following formula (Eq. 2):

$$
\mathrm{BCF}=\mathrm{C}_{\mathrm{Root}} / \mathrm{C}_{\text {Soil }}
$$

where $\mathrm{C}_{\text {Root }}$ is the concentration of an $\mathrm{HM}$ in the root $\left(\mathrm{mg} \mathrm{kg}^{-1}\right)$ and $\mathrm{C}_{\text {Soil }}$ is its concentration in the soil $\left(\mathrm{mg} \mathrm{kg}^{-1}\right)$. In addition, the TF determines the ability of $P$. vulgaris to translocate an HM from the root to each of the stem, leaf and pod (Eid and Shaltout, 2016) and is calculated using the following formulas (Eqs. 3, 4 and 5):

$$
\begin{aligned}
& \mathrm{TF}_{\text {Stem }}=\mathrm{C}_{\text {Stem }} / \mathrm{C}_{\text {Root }} \\
& \mathrm{TF}_{\text {Leaf }}=\mathrm{C}_{\text {Leaf }} / \mathrm{C}_{\text {Root }} \\
& \mathrm{TF}_{\text {Pod }}=\mathrm{C}_{\text {Pod }} / \mathrm{C}_{\text {Root }}
\end{aligned}
$$


where $\mathrm{C}_{\text {Root}}, \mathrm{C}_{\text {Stem }}, \mathrm{C}_{\text {Leaf }}$ and $\mathrm{C}_{\text {Pod }}$ are the concentrations of an $\mathrm{HM}$ in these tissues (mg $\mathrm{kg}^{-1}$ ). The BCFs and TFs data were subjected to an ANOVA-1 to evaluate the differences among the ten HMs. Significant difference between means among the ten HMs were identified using the Tukey's HSD test at $p<0.05$. Pearson's simple linear correlation coefficient $(r)$ was estimated for the BCF of a certain HM in the plant tissues with soil $\mathrm{pH}$ and soil $\mathrm{OM}$. The same coefficient was determined to assess the relationship between the plant HMs and soil HMs, $\mathrm{pH}$ and OM.

Eighteen observations from the dataset for each of the root, stem and leaf were selected randomly as a validation dataset. The other eighteen observations were used to establish the regression models for predicting the HM contents in P. vulgaris tissues, based on some soil variables used as independent variables ( $\mathrm{pH}, \mathrm{OM}, \mathrm{Cd}, \mathrm{Co}, \mathrm{Cr}, \mathrm{Cu}$, $\mathrm{Fe}, \mathrm{Mn}, \mathrm{Mo}, \mathrm{Ni}, \mathrm{Pb}$ and $\mathrm{Zn}$ ). The number of individuals that produced pods was twelve (in treatments with concentrations of 10 and $20 \mathrm{~g} \mathrm{~kg}^{-1}$ only); thus, six observations were used as a validation dataset, while the other six observations were used in the regression procedure. Following Novotná et al. (2015) and Yang et al. (2013), the OM content, pH value and HM content of the soil are considered the most important factors with which to explain plant HM concentrations. The general equation of the model was established using the following formula (Eq. 6):

$$
\mathrm{C}_{\text {Plant }}=a+b \times \mathrm{C}_{\text {Soil }}+c \times \mathrm{pH}+d \times \mathrm{OM}
$$

where $\mathrm{C}_{\text {Plant }}$ is the concentration of a given $\mathrm{HM}$ in any $P$. vulgaris tissue; $\mathrm{C}_{\text {Soil }}$ is its concentration in the soil; $\mathrm{OM}$ is the soil organic matter content (\%); and $a, b, c$ and $d$ are the regression coefficients.

Model quality was evaluated using the coefficient of determination $\left(R^{2}\right)$, model efficiency $(M E)$, model strength (MNAE) and model bias $(M N B)$ using the formulas by Novotná et al. (2015) (Eqs. 7, 8 and 9):

$$
\begin{gathered}
M E=1-\left(\sum\left(\mathrm{C}_{\text {Model }}-\mathrm{C}_{\text {Measured }}\right)^{2} / \sum\left(\mathrm{C}_{\text {Measured }}-\mathrm{C}_{\text {Mean }}\right)^{2}\right) \\
M N A E=\left(\sum\left(\left|\mathrm{C}_{\text {Model }}-\mathrm{C}_{\text {Measured }}\right| / \mathrm{C}_{\text {Measured }}\right)\right) / \mathrm{n} \\
M N B=\sum\left(\mathrm{C}_{\text {Model }}-\mathrm{C}_{\text {Measured }}\right) / \sum \mathrm{C}_{\text {Measured }}
\end{gathered}
$$

where $\mathrm{C}_{\text {Model }}$ is the predicted concentration of an $\mathrm{HM}$ given by the model, $\mathrm{C}_{\text {Measured }}$ is the actual $\mathrm{HM}$ concentration, $\mathrm{C}_{\text {Mean }}$ is the mean of the actual $\mathrm{HM}$ concentrations, and $n$ is the number of observations. Student's $t$-test was applied to assess the significance of the difference between the predicted concentration of an HM in a tissue and its actual value in the same tissue. All these analyses were carried out in SPSS 15.0 (SPSS, 2006).

\section{Results}

For prior testing of the quality of the used agricultural soil and SS, the OM, pH value, and concentrations of ten common HMs were analyzed. The data presented in Appendix 1 show that the agricultural soil from the study region is poor in fertility because it contains less than $1 \% \mathrm{OM}$. The $\mathrm{pH}$ of this soil is 8.7 (basic), which reduces the availability of most nutrients (Sharma et al., 2017). The HM concentrations in the agricultural soil ranged between a minimum of $1.1 \mathrm{mg} \mathrm{kg}^{-1}$ for Mo and a maximum of 
42,400 mg kg-1 for Fe; while in the SS they have a range of $0.9 \mathrm{mg} \mathrm{kg}^{-1}$ for Mo and $24,100 \mathrm{mg} \mathrm{kg}^{-1}$ for Fe (Appendix 1). In addition, the concentration of $5 \mathrm{HMs}(\mathrm{Cd}, \mathrm{Co}$, $\mathrm{Fe}, \mathrm{Mn}$ and Mo) are higher in the agricultural soil than in the SS, while the reverse is true regarding the other $5 \mathrm{HMs}$.

After plant harvesting, the $\mathrm{pH}$ values of the soil-sludge mixture were alkaline, with a range of $6.8-8.5$, a mean of 7.5 and a coefficient of variance $(\mathrm{CV})$ of $7 \%$; the $\mathrm{OM}$ ranged between 1 and $9 \%$, with a mean of $5 \%$ and a $\mathrm{CV}$ of $47 \%$ (Table 1). The concentrations of the ten $\mathrm{HMs}$ in the soil descended as follows: $\mathrm{Fe}>\mathrm{Mn}>\mathrm{Cr}>\mathrm{Zn}>\mathrm{Ni}>\mathrm{Co}>\mathrm{Cu}>\mathrm{Pb}>\mathrm{Cd}>\mathrm{Mo}$. Iron had the highest mean concentration of $41,878 \mathrm{mg} \mathrm{kg}^{-1}$, while Mo had the lowest mean concentration of $1 \mathrm{mg}$ $\mathrm{kg}^{-1}$. Regarding the plant tissues, most of the HM concentrations were lower in the pods than in the other tissues, except Co, Mo and Ni (Table 2). In contrast, the HMs were more abundant in the roots than in all the areal tissues, except Mo. In descending order, the ten $\mathrm{HMs}$ in the three areal tissues were $\mathrm{Fe}>\mathrm{Mn}>\mathrm{Zn}>\mathrm{Mo}>\mathrm{Ni}>\mathrm{Cu}>\mathrm{Cr}>\mathrm{Co}>\mathrm{Pb}>\mathrm{Cd}$, while those in the roots were $\mathrm{Fe}>\mathrm{Mn}>\mathrm{Zn}>\mathrm{Cr}>\mathrm{Cu}>\mathrm{Ni}>\mathrm{Co}>\mathrm{Mo}>\mathrm{Pb}>\mathrm{Cd}$.

Table 1. Chemical characteristics of the soil $(n=36)$ amended with sewage sludge after harvesting Phaseolus vulgaris plants grown for 57 days

\begin{tabular}{c|c|c|c|c|c|c|c|c|c|c|c|c}
\hline \multirow{2}{*}{ Value } & \multirow{2}{*}{$\mathbf{p H}$} & \multirow{2}{*}{ OM (\%) } & \multicolumn{10}{c}{ Heavy metal concentration $\left(\mathbf{m g ~ k g}^{-\mathbf{1}}\right)$} \\
\cline { 4 - 12 } & & & $\mathbf{C d}$ & $\mathbf{C o}$ & $\mathbf{C r}$ & $\mathbf{C u}$ & $\mathbf{F e}$ & $\mathbf{M n}$ & $\mathbf{M o}$ & $\mathbf{N i}$ & $\mathbf{P b}$ & $\mathbf{Z n}$ \\
\hline Minimum & 6.8 & 1 & 2 & 24 & 123 & 15 & 23,488 & 557 & 1 & 29 & 3 & 64 \\
Maximum & 8.5 & 9 & 4 & 49 & 159 & 39 & 67,273 & 796 & 2 & 44 & 5 & 120 \\
Mean & $\mathbf{7 . 5}$ & $\mathbf{5}$ & $\mathbf{3}$ & $\mathbf{2 9}$ & $\mathbf{1 4 1}$ & $\mathbf{2 8}$ & $\mathbf{4 1 , 8 7 8}$ & $\mathbf{6 2 3}$ & $\mathbf{1}$ & $\mathbf{3 4}$ & $\mathbf{4}$ & $\mathbf{9 5}$ \\
$\mathrm{CV}(\%)$ & 7 & 47 & 18 & 17 & 7 & 28 & 16 & 8 & 14 & 10 & 20 & 21 \\
\hline
\end{tabular}

$\mathrm{CV}$ : coefficient of variance, OM: organic matter content

Table 2. Heavy metal concentrations in pods, leaves, stems and roots of Phaseolus vulgaris plants harvested after 57 days

\begin{tabular}{|c|c|c|c|c|c|c|c|c|c|c|c|}
\hline \multirow{2}{*}{ Tissue } & \multirow{2}{*}{ Value } & \multicolumn{10}{|c|}{ Heavy metal concentration $\left(\mathrm{mg} \mathrm{kg}^{-1}\right)$} \\
\hline & & Cd & Co & $\mathrm{Cr}$ & $\mathbf{C u}$ & $\mathbf{F e}$ & Mn & Mo & $\mathbf{N i}$ & $\mathbf{P b}$ & $\mathbf{Z n}$ \\
\hline \multirow{4}{*}{ Pod } & Minimum & 0.1 & 1 & 1 & 7 & 118 & 25 & 12 & 7 & 0.1 & 21 \\
\hline & Maximum & 0.3 & 2 & 2 & 11 & 165 & 38 & 17 & 14 & 1 & 29 \\
\hline & Mean $(n=12)$ & $0.2 \mathrm{a}$ & $2 \mathrm{a}$ & $2 \mathrm{a}$ & 9a & $147 \mathrm{a}$ & $32 \mathrm{a}$ & $14 \mathrm{~b}$ & $11 b$ & $0.3 \mathrm{a}$ & $26 a$ \\
\hline & $\mathrm{CV}(\%)$ & 30 & 9 & 21 & 17 & 12 & 15 & 13 & 29 & 83 & 13 \\
\hline \multirow{4}{*}{ Leaf } & Minimum & 0.1 & 1 & 2 & 7 & 183 & 138 & 7 & 3 & 0.2 & 20 \\
\hline & Maximum & 0.4 & 2 & 5 & 13 & 1,230 & 228 & 20 & 9 & 2 & 33 \\
\hline & Mean $(n=36)$ & $0.2 \mathrm{a}$ & $1 \mathrm{a}$ & $3 a$ & $10 \mathrm{a}$ & $389 a$ & $187 \mathrm{~b}$ & $15 \mathrm{~b}$ & $5 \mathrm{a}$ & $1 \mathrm{a}$ & $27 \mathrm{a}$ \\
\hline & $\mathrm{CV}(\%)$ & 30 & 38 & 35 & 17 & 78 & 16 & 26 & 30 & 53 & 15 \\
\hline \multirow{4}{*}{ Stem } & Minimum & 0.2 & 1 & 1 & 8 & 85 & 24 & 10 & 3 & 1 & 19 \\
\hline & Maximum & 1 & 2 & 6 & 18 & 734 & 191 & 48 & 8 & 4 & 60 \\
\hline & Mean $(n=36)$ & $0.4 \mathrm{~b}$ & $1 \mathrm{a}$ & $3 a$ & $12 \mathrm{a}$ & $272 \mathrm{a}$ & $76 \mathrm{a}$ & 29c & $5 \mathrm{a}$ & $1 \mathrm{a}$ & $34 \mathrm{a}$ \\
\hline & CV $(\%)$ & 40 & 40 & 55 & 19 & 78 & 73 & 41 & 24 & 57 & 34 \\
\hline \multirow{4}{*}{ Root } & Minimum & 0.2 & 6 & 19 & 2 & 4,078 & 258 & 2 & 11 & 2 & 53 \\
\hline & Maximum & 2 & 16 & 67 & 46 & 17,760 & 1190 & 15 & 27 & 9 & 222 \\
\hline & Mean $(n=36)$ & 1c & $11 b$ & $39 b$ & $27 \mathrm{~b}$ & $10,283 b$ & $673 c$ & $7 \mathrm{a}$ & $18 \mathrm{c}$ & $4 b$ & $126 \mathrm{~b}$ \\
\hline & CV $(\%)$ & 52 & 24 & 38 & 47 & 41 & 42 & 50 & 26 & 53 & 50 \\
\hline \multicolumn{2}{|r|}{$F$-value } & $47.4 * * *$ & $346.9^{* * *}$ & $167.9^{* * *}$ & $44.7 * * *$ & $155.5^{* * *} *$ & $105.1 * * *$ & $55.9^{* * * *}$ & $146.7 * * *$ & $56.5^{* * *}$ & $64.3^{* * * *}$ \\
\hline
\end{tabular}

$F$-values represent one-way ANOVA, degrees of freedom $=3$. Means in the same column followed by different letters are significantly different at $p<0.05$ according to Tukey's HSD test. $* * *: p<0.001, \mathrm{CV}$ : coefficient of variance 
The BCFs and TFs were < 1 for most HMs, except the BCFs of Mn (1.07), Mo (6.12), $\mathrm{Pb}(1.03)$ and $\mathrm{Zn}(1.25)$ and the TFs of Mo (2.53-4.57) in the three areal tissues (Table 3). In addition, the BCFs and TFs varied among the studied HMs (Table 3). Correlations were significant and negative between the BCFs of all ten HMs and soil $\mathrm{pH}$, with the highest $r$-value of 0.89 for $\mathrm{Mn}$ and the lowest of 0.37 for $\mathrm{Pb}$ (Fig. 1), but significant and positive between the BCFs and soil OM, with a maximum $r$-value of 0.84 for $\mathrm{Mn}$ and a minimum of 0.36 for $\mathrm{Pb}$ (Fig. 2).

Table 3. Descriptive statistical results (mean \pm standard error) of the bioconcentration factors (BCFs) of heavy metals from soil to Phaseolus vulgaris roots and the translocation factors of heavy metals from Phaseolus vulgaris roots to stems $\left(T F_{\text {stem }}\right)$, leaves $\left(T F_{\text {leaf }}\right)$ and pods $\left(T F_{p o d}\right)$

\begin{tabular}{c|c|c|c|c}
\hline Heavy metal & BCF $(\boldsymbol{n}=\mathbf{3 6})$ & $\mathbf{T F}_{\text {stem }}(\boldsymbol{n}=\mathbf{3 6})$ & $\mathbf{T F}_{\text {leaf }}(\boldsymbol{n}=\mathbf{3 6})$ & $\mathbf{T F}_{\boldsymbol{p o d}}(\boldsymbol{n}=\mathbf{1 2})$ \\
\hline $\mathrm{Cd}$ & $0.29 \pm 0.02 \mathrm{ab}$ & $0.49 \pm 0.03 \mathrm{ab}$ & $0.27 \pm 0.02 \mathrm{a}$ & $0.27 \pm 0.03 \mathrm{a}$ \\
$\mathrm{Co}$ & $0.38 \pm 0.01 \mathrm{ab}$ & $0.11 \pm 0.01 \mathrm{a}$ & $0.12 \pm 0.01 \mathrm{a}$ & $0.17 \pm 0.01 \mathrm{a}$ \\
$\mathrm{Cr}$ & $0.27 \pm 0.02 \mathrm{ab}$ & $0.07 \pm 0.00 \mathrm{a}$ & $0.07 \pm 0.00 \mathrm{a}$ & $0.05 \pm 0.00 \mathrm{a}$ \\
$\mathrm{Cu}$ & $0.92 \pm 0.05 \mathrm{bcd}$ & $0.83 \pm 0.21 \mathrm{~b}$ & $0.63 \pm 0.14 \mathrm{~b}$ & $0.42 \pm 0.02 \mathrm{a}$ \\
$\mathrm{Fe}$ & $0.25 \pm 0.02 \mathrm{a}$ & $0.03 \pm 0.00 \mathrm{a}$ & $0.04 \pm 0.00 \mathrm{a}$ & $0.02 \pm 0.00 \mathrm{a}$ \\
$\mathrm{Mn}$ & $1.07 \pm 0.07 \mathrm{~cd}$ & $0.10 \pm 0.01 \mathrm{a}$ & $0.32 \pm 0.02 \mathrm{a}$ & $0.06 \pm 0.00 \mathrm{a}$ \\
$\mathrm{Mo}$ & $6.12 \pm 0.47 \mathrm{e}$ & $4.57 \pm 0.37 \mathrm{c}$ & $2.53 \pm 0.21 \mathrm{c}$ & $3.84 \pm 0.73 \mathrm{~b}$ \\
$\mathrm{Ni}$ & $0.53 \pm 0.02 \mathrm{abc}$ & $0.31 \pm 0.01 \mathrm{ab}$ & $0.29 \pm 0.01 \mathrm{a}$ & $0.77 \pm 0.05 \mathrm{a}$ \\
$\mathrm{Pb}$ & $1.03 \pm 0.08 \mathrm{~cd}$ & $0.32 \pm 0.03 \mathrm{ab}$ & $0.21 \pm 0.02 \mathrm{a}$ & $0.11 \pm 0.02 \mathrm{a}$ \\
$\mathrm{Zn}$ & $1.25 \pm 0.07 \mathrm{~d}$ & $0.30 \pm 0.01 \mathrm{ab}$ & $0.26 \pm 0.02 \mathrm{a}$ & $0.35 \pm 0.02 \mathrm{a}$ \\
\hline$F$-value & $128.7 * * *$ & $101.8 * * *$ & $83.4 * * *$ & $24.7 * * *$ \\
\hline
\end{tabular}

$F$-values represent one-way ANOVA, degrees of freedom $=9$. Means in the same column followed by different letters are significantly different at $p<0.05$ according to Tukey's HSD test. $* * *: p<0.001$

With a few exceptions, the correlation between an HM in the four tissues and the same HM in the soil was significant and positive (Table 4). In addition, significant negative correlations were detected between soil $\mathrm{pH}$ and only some HMs in the pods ( $r=-0.76$ with $\mathrm{Co},-0.67$ with $\mathrm{Mo},-0.89$ with $\mathrm{Ni}$ and -0.81 with $\mathrm{Zn}, p<0.001)$ but between soil $\mathrm{pH}$ and all HMs in the other three tissues. In contrast, soil OM showed the reverse trend with some HMs in the pods and all HMs in the other three tissues.

Correlations between the measured and predicted HM values, with high $R^{2}$, high $M E s$ and low mean MNAEs, reflected the goodness of fit of the model. Additionally, the presence of a non-significant difference ( $p$-value) between the measured and predicted concentrations of the HMs indicated good performance of the developed model. For the pods, the regression equation that had the highest $R^{2}(0.99)$ and highest $M E$ (1.00) but the lowest $M N B$ (0.01) was that of Co. For the leaves, the equation with the highest $R^{2}$ (0.90) and highest $M E(0.92)$ but the lowest $M N B$ (0.001) was that of Mo. Similar results were obtained for Mo in the stems and Mn in the roots (Table 5). Applying a $t$ test that assessed the significance of the differences between the actual and predicted values of the ten HMs in the four tissues revealed that the differences were nonsignificant $(p>0.05)$.

\section{Discussion}

The soil-sludge combination supporting $P$. vulgaris was slightly alkaline ( $\mathrm{pH}=7.5)$. High soil $\mathrm{pH}$ is known to enhance the adsorption of many HMs, thus decreasing their solubility in soil solutions (Chaudri et al., 2007; Zeng et al., 2011). The adsorption and 
desorption properties of soil are among the factors affecting the mobility and availability of HMs (Krishnamurti et al., 1999). Both properties are associated with soil $\mathrm{pH}, \mathrm{OM}$, clay minerals and oxidation-reduction status (Antoniadis et al., 2008; Usman et al., 2008). Significant negative correlations were detected between soil $\mathrm{pH}$ and most of the estimated HM concentrations in the four tissues of $P$. vulgaris. Zhao et al. (2010) reported that this soil factor plays a vital role in determining the solubility and availability of soil HMs.
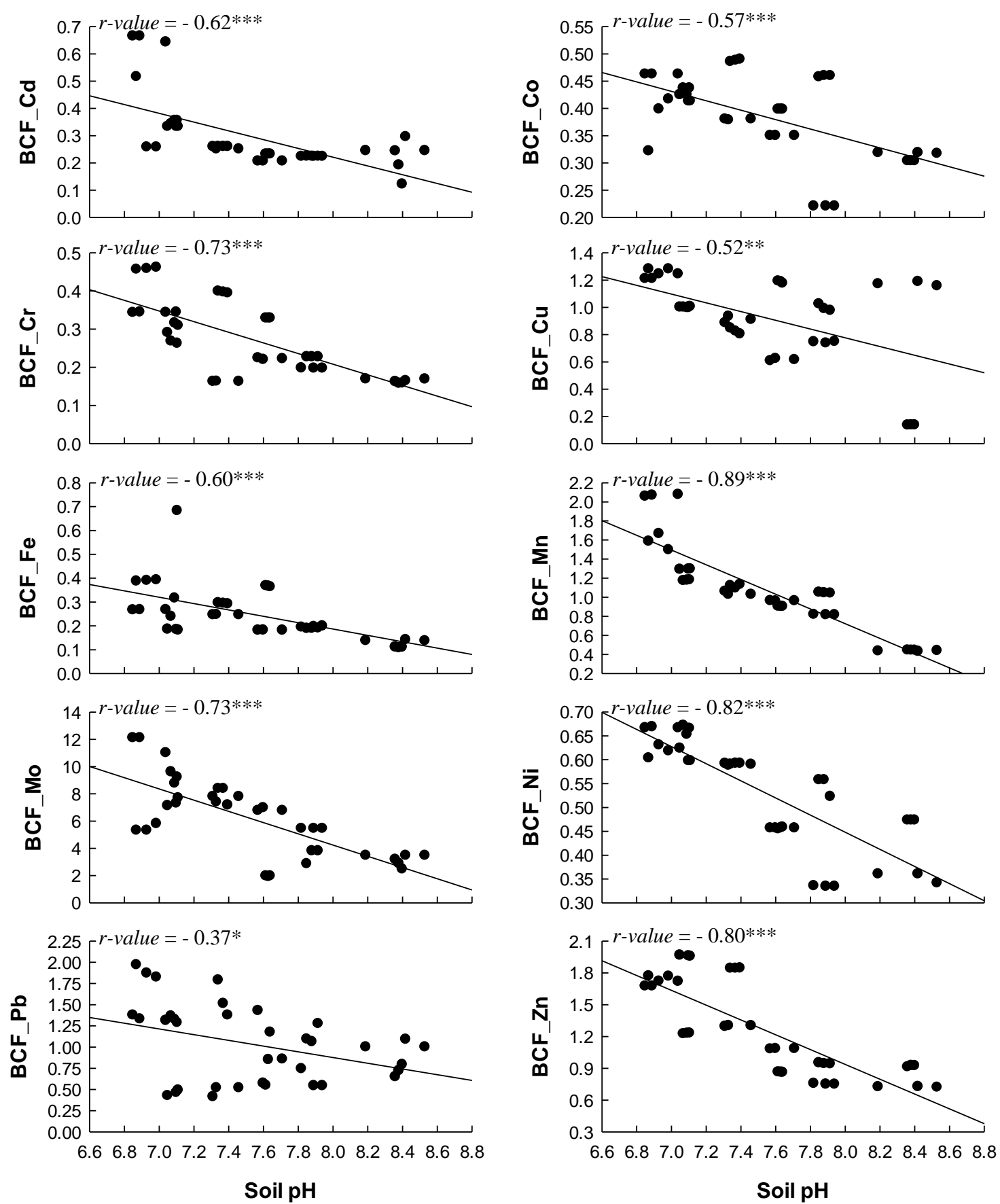

Figure 1. Linear relationships ( $r$-values, $n=36$ ) between bioconcentration factors (BCFs) of heavy metals in Phaseolus vulgaris roots and soil $p H . *: p<0.05, * *: p<0.01$, ***:

$$
p<0.001
$$



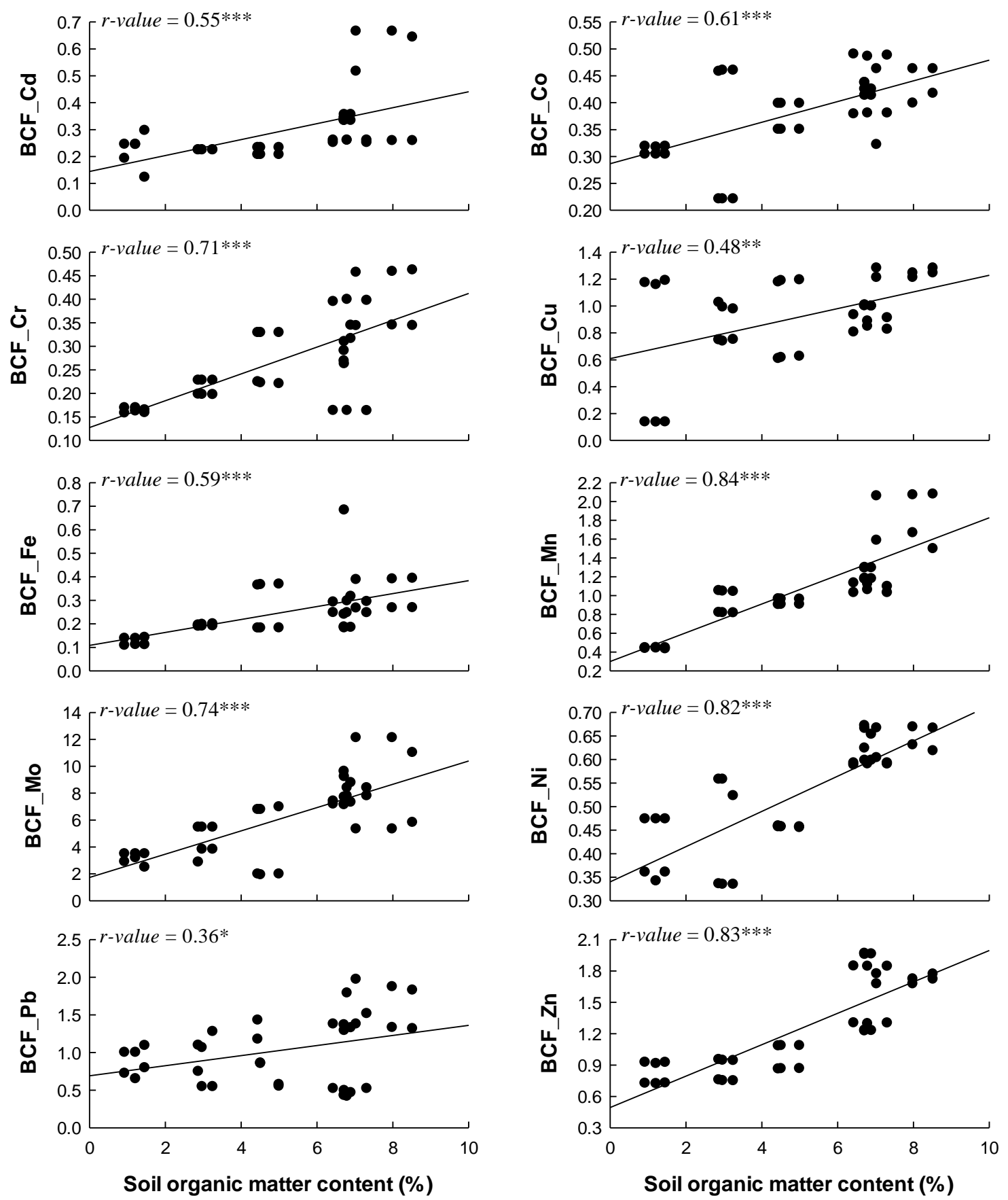

Figure 2. Linear relationships ( $r$-values, $n=36$ ) between bioconcentration factors (BCFs) of heavy metals in Phaseolus vulgaris roots and soil organic matter content. *: $p<0.05, * *$ : $p<0.01, * * *: p<0.001$

In contrast, soil OM had significant positive correlations with most of the estimated HM concentrations in the tissues of the studied species. OM content plays an important role in determining the availability and mobility of HMs in soils, and it helps provide organic chemicals to the soil solution that act as chelates to increase HM availability to plants (Zeng et al., 2011). Decomposition of soil OM forms soluble organic-HM complexes and consequently more bioavailable HMs (Antoniadis and 
Alloway, 2002). Therefore, SS must be applied carefully under appropriate conditions to reduce the accumulation of HMs in soil and consequently their transfer to plant tissues.

Table 4. Pearson correlation coefficient ( $r$-value) between heavy metals in Phaseolus vulgaris tissues and their concentrations in the soil

\begin{tabular}{|c|c|c|c|c|c|c|c|c|c|c|c|c|}
\hline \multirow{2}{*}{$\begin{array}{c}\text { P. vulgaris } \\
\text { heavy metals }\end{array}$} & \multicolumn{10}{|c|}{ Soil heavy metals } & \multirow{2}{*}{ Soil pH } & \multirow{2}{*}{ Soil OM } \\
\hline & Cd & Co & $\mathrm{Cr}$ & $\mathbf{C u}$ & $\mathrm{Fe}$ & Mn & Mo & $\mathbf{N i}$ & $\mathbf{P b}$ & $\mathbf{Z n}$ & & \\
\hline \multicolumn{13}{|l|}{$\operatorname{Pod}(n=12)$} \\
\hline $\mathrm{Cd}$ & $\underline{-0.33}$ & $\underline{-0.36}$ & $\underline{0.09}$ & $\underline{0.10}$ & $\underline{-0.36}$ & $\underline{-0.29}$ & $\underline{-0.10}$ & $\underline{-0.11}$ & $\underline{-0.09}$ & $\underline{-0.16}$ & $\underline{-0.19}$ & $\underline{0.43}$ \\
\hline Co & $\underline{0.28}$ & 0.83 & $\underline{0.08}$ & 0.83 & 0.72 & $\underline{0.25}$ & 0.89 & $\underline{0.02}$ & 0.96 & 0.94 & -0.76 & 0.77 \\
\hline $\mathrm{Cr}$ & -0.48 & $\underline{0.50}$ & $\underline{-0.53}$ & $\underline{0.29}$ & 0.67 & -0.51 & $\underline{0.53}$ & -0.66 & $\underline{0.47}$ & $\underline{0.40}$ & $\underline{-0.51}$ & $\underline{0.54}$ \\
\hline $\mathrm{Cu}$ & $\underline{0.15}$ & -0.52 & 0.74 & $\underline{0.48}$ & -0.68 & $\underline{0.19}$ & $\underline{-0.09}$ & $\underline{0.51}$ & $\underline{0.03}$ & $\underline{0.01}$ & $\underline{-0.54}$ & $\underline{0.55}$ \\
\hline $\mathrm{Fe}$ & $\underline{-0.52}$ & $\underline{0.52}$ & -0.59 & $\underline{0.26}$ & 0.70 & $\underline{-0.55}$ & $\underline{0.56}$ & -0.71 & $\underline{0.49}$ & $\underline{0.38}$ & $\underline{-0.51}$ & $\underline{0.52}$ \\
\hline $\mathrm{Mn}$ & 0.83 & $\underline{-0.20}$ & 0.96 & $\underline{0.43}$ & $\underline{-0.53}$ & 0.86 & $\underline{-0.07}$ & 0.99 & $\underline{0.10}$ & $\underline{0.21}$ & $\underline{-0.11}$ & $\underline{0.11}$ \\
\hline Mo & $\underline{0.28}$ & $\underline{-0.31}$ & 0.80 & 0.67 & $\underline{-0.52}$ & $\underline{0.32}$ & $\underline{0.11}$ & 0.58 & $\underline{0.25}$ & $\underline{0.24}$ & -0.67 & 0.69 \\
\hline $\mathrm{Ni}$ & -0.23 & $\underline{0.32}$ & $\underline{0.00}$ & 0.68 & $\underline{0.33}$ & $\underline{-0.24}$ & 0.60 & $\underline{-0.23}$ & 0.64 & $\underline{0.54}$ & -0.89 & 0.90 \\
\hline $\mathrm{Pb}$ & $\underline{-0.20}$ & $\underline{-0.32}$ & $\underline{0.25}$ & $\underline{0.29}$ & -0.32 & $\underline{-0.16}$ & $\underline{0.11}$ & $\underline{0.03}$ & $\underline{-0.05}$ & $\underline{-0.03}$ & $\underline{-0.33}$ & $\underline{0.38}$ \\
\hline $\mathrm{Zn}$ & 0.58 & $\underline{0.28}$ & 0.76 & 0.97 & $\underline{-0.01}$ & 0.59 & $\underline{0.56}$ & 0.63 & 0.73 & 0.75 & -0.81 & 0.84 \\
\hline \multicolumn{13}{|l|}{ Leaf $(n=36)$} \\
\hline $\mathrm{Cd}$ & 0.54 & 0.70 & $\underline{0.22}$ & 0.48 & $\underline{0.24}$ & 0.53 & 0.54 & 0.57 & 0.45 & 0.45 & -0.67 & 0.63 \\
\hline Co & 0.66 & 0.78 & 0.44 & 0.75 & 0.36 & 0.64 & 0.59 & 0.77 & 0.81 & 0.83 & -0.85 & 0.81 \\
\hline $\mathrm{Cr}$ & 0.56 & 0.71 & 0.35 & 0.56 & $\underline{0.16}$ & 0.61 & 0.69 & 0.80 & 0.64 & 0.62 & -0.76 & 0.74 \\
\hline $\mathrm{Cu}$ & 0.66 & $\underline{0.31}$ & 0.73 & 0.82 & 0.46 & 0.33 & 0.47 & 0.54 & 0.74 & 0.61 & -0.89 & 0.86 \\
\hline $\mathrm{Fe}$ & 0.46 & 0.74 & $\underline{0.22}$ & 0.53 & $\underline{0.23}$ & 0.64 & 0.65 & 0.80 & 0.67 & 0.55 & -0.64 & 0.60 \\
\hline $\mathrm{Mn}$ & 0.76 & 0.55 & 0.40 & 0.75 & $\underline{0.21}$ & 0.46 & 0.62 & 0.63 & 0.72 & 0.80 & -0.85 & 0.86 \\
\hline Mo & 0.72 & 0.47 & 0.72 & 0.80 & 0.36 & 0.42 & 0.75 & 0.62 & 0.83 & 0.82 & -0.87 & 0.88 \\
\hline $\mathrm{Ni}$ & 0.57 & 0.69 & 0.45 & 0.78 & 0.47 & 0.48 & 0.59 & 0.62 & 0.82 & 0.76 & -0.85 & 0.80 \\
\hline $\mathrm{Pb}$ & 0.42 & $\underline{0.11}$ & 0.68 & 0.66 & 0.44 & $\underline{0.16}$ & 0.35 & 0.38 & 0.69 & 0.47 & -0.72 & 0.66 \\
\hline $\mathrm{Zn}$ & 0.75 & 0.53 & 0.53 & 0.80 & $\underline{0.32}$ & 0.48 & 0.57 & 0.63 & 0.88 & 0.78 & -0.94 & 0.88 \\
\hline \multicolumn{13}{|l|}{ Stem $(n=36)$} \\
\hline $\mathrm{Cd}$ & 0.51 & $\underline{0.24}$ & 0.54 & 0.50 & $\underline{0.21}$ & $\underline{0.28}$ & $\underline{0.29}$ & 0.40 & 0.44 & 0.59 & -0.70 & 0.69 \\
\hline Co & 0.60 & 0.46 & 0.35 & 0.65 & $\underline{0.15}$ & 0.42 & 0.52 & 0.60 & 0.78 & 0.71 & -0.81 & 0.78 \\
\hline $\mathrm{Cr}$ & 0.55 & 0.58 & 0.52 & 0.76 & 0.44 & 0.48 & 0.61 & 0.69 & 0.77 & 0.64 & -0.80 & 0.78 \\
\hline $\mathrm{Cu}$ & 0.61 & 0.80 & 0.41 & 0.70 & 0.34 & 0.70 & 0.42 & 0.81 & 0.55 & 0.68 & -0.81 & 0.81 \\
\hline $\mathrm{Fe}$ & 0.45 & 0.48 & 0.46 & 0.68 & 0.42 & 0.36 & 0.55 & 0.59 & 0.74 & 0.58 & -0.74 & 0.70 \\
\hline $\mathrm{Mn}$ & 0.49 & 0.54 & 0.56 & 0.76 & 0.47 & 0.43 & 0.55 & 0.65 & 0.75 & 0.65 & -0.79 & 0.78 \\
\hline Mo & 0.81 & 0.67 & 0.47 & 0.83 & $\underline{0.28}$ & 0.61 & 0.63 & 0.75 & 0.81 & 0.83 & -0.90 & 0.91 \\
\hline $\mathrm{Ni}$ & 0.37 & 0.45 & 0.53 & 0.62 & 0.39 & 0.38 & $\underline{0.19}$ & 0.59 & 0.50 & 0.50 & -0.75 & 0.75 \\
\hline $\mathrm{Pb}$ & 0.48 & 0.76 & $\underline{0.28}$ & 0.56 & $\underline{0.25}$ & 0.71 & 0.71 & 0.82 & 0.65 & 0.53 & -0.64 & 0.63 \\
\hline $\mathrm{Zn}$ & 0.68 & 0.82 & 0.37 & 0.71 & $\underline{0.27}$ & 0.74 & 0.60 & 0.84 & 0.72 & 0.72 & -0.82 & 0.81 \\
\hline \multicolumn{13}{|l|}{ Root $(n=36)$} \\
\hline $\mathrm{Cd}$ & 0.54 & $\underline{0.31}$ & 0.64 & 0.69 & 0.42 & $\underline{0.16}$ & 0.51 & 0.40 & 0.79 & 0.67 & -0.77 & 0.70 \\
\hline Co & 0.46 & 0.64 & $\underline{0.26}$ & 0.56 & $\underline{0.29}$ & 0.35 & 0.49 & 0.51 & 0.56 & 0.64 & -0.78 & 0.78 \\
\hline $\mathrm{Cr}$ & 0.58 & 0.49 & 0.48 & 0.54 & $\underline{0.21}$ & 0.46 & 0.52 & 0.68 & 0.49 & 0.59 & -0.78 & 0.76 \\
\hline $\mathrm{Cu}$ & 0.66 & 0.42 & 0.72 & 0.86 & 0.43 & 0.43 & 0.72 & 0.62 & 0.84 & 0.66 & -0.83 & 0.81 \\
\hline $\mathrm{Fe}$ & 0.56 & 0.44 & 0.70 & 0.69 & 0.40 & 0.48 & 0.38 & 0.63 & 0.45 & 0.50 & -0.76 & 0.76 \\
\hline $\mathrm{Mn}$ & 0.71 & 0.57 & 0.54 & 0.79 & 0.42 & 0.47 & 0.59 & 0.65 & 0.82 & 0.77 & -0.92 & 0.87 \\
\hline Mo & 0.64 & $\underline{0.28}$ & 0.67 & 0.76 & 0.42 & $\underline{0.18}$ & 0.48 & 0.35 & 0.80 & 0.83 & -0.79 & 0.80 \\
\hline $\mathrm{Ni}$ & 0.55 & 0.64 & 0.48 & 0.73 & 0.39 & 0.48 & 0.47 & 0.68 & 0.70 & 0.70 & -0.89 & 0.89 \\
\hline $\mathrm{Pb}$ & 0.33 & 0.56 & 0.39 & 0.44 & 0.35 & 0.39 & 0.50 & 0.56 & 0.43 & 0.50 & -0.59 & 0.56 \\
\hline $\mathrm{Zn}$ & 0.66 & 0.56 & 0.44 & 0.73 & $\underline{0.27}$ & 0.42 & 0.58 & 0.61 & 0.80 & 0.87 & -0.86 & 0.87 \\
\hline
\end{tabular}

OM: organic matter content. $n=12: p<0.05(r \geq 0.58), p<0.01 \quad(r \geq 0.72), p<0.001 \quad(r \geq 0.86) . n=36: p<0.05 \quad(r \geq 0.33)$, $p<0.01(r \geq 0.43), p<0.001(r \geq 0.55)$. Nonsignificant $r$ values $(p>0.05)$ are underlined 

mathematical model

$$
-7031 \text { - }
$$

Table 5. Regression models between heavy metal concentrations in Phaseolus vulgaris tissues $\left(\mathrm{mg} \mathrm{kg}^{-1}\right)$ and soil heavy metals $\left(\mathrm{mg} \mathrm{kg}^{-1}\right), \mathrm{pH}$ and organic matter (OM) content (\%)

\begin{tabular}{|c|c|c|c|c|c|c|}
\hline \multirow{2}{*}{ Equation } & \multirow{2}{*}{$R^{2}$} & \multirow{2}{*}{$M E$} & \multirow{2}{*}{ MNAE } & \multirow{2}{*}{$M N B$} & \multicolumn{2}{|c|}{ Student's $t$-test } \\
\hline & & & & & $t$-value & $p$ \\
\hline \multicolumn{7}{|l|}{ Pod } \\
\hline $\mathrm{Cd}_{\text {pod }}=-3.576-0.054 \times \mathrm{Cd}_{\text {soil }}+0.460 \times \mathrm{pH}+0.099 \times \mathrm{OM}$ & 0.54 & 0.98 & 0.21 & 0.00 & 0.02 & 0.98 \\
\hline $\mathrm{Co}_{\text {pod }}=2.317+0.025 \times \mathrm{Co}_{\text {soil }}-0.217 \times \mathrm{pH}+0.052 \times \mathrm{OM}$ & 0.99 & 1.00 & 0.01 & 0.01 & 1.56 & 0.18 \\
\hline $\mathrm{Cr}_{p o d}=8.114-0.036 \times \mathrm{Cr}_{\text {soil }}-0.347 \times \mathrm{pH}+0.274 \times \mathrm{OM}$ & 0.89 & 0.99 & 0.07 & 0.07 & 2.59 & 0.12 \\
\hline $\mathrm{Cu}_{\text {pod }}=23.505-0.010 \times \mathrm{Cu}_{\text {soil }}-2.686 \times \mathrm{pH}+0.611 \times \mathrm{OM}$ & 0.32 & 0.89 & 0.18 & 0.12 & 1.67 & 0.16 \\
\hline $\mathrm{Fe}_{\text {pod }}=-191.906+0.008 \times \mathrm{Fe}_{\text {soil }}-0.254 \times \mathrm{pH}+8.584 \times \mathrm{OM}$ & 0.66 & 0.99 & 0.13 & 0.12 & 1.69 & 0.23 \\
\hline $\mathrm{Mn}_{\text {pod }}=-21.811+0.107 \times \mathrm{Mn}_{\text {soil }}-1.444 \times \mathrm{pH}-0.176 \times \mathrm{OM}$ & 0.74 & 0.99 & 0.07 & 0.01 & 0.30 & 0.78 \\
\hline $\mathrm{Mo}_{\text {pod }}=99.371-34.628 \times \mathrm{Mo}_{\text {soil }}-6.639 \times \mathrm{pH}-1.001 \times \mathrm{OM}$ & 0.62 & 0.99 & 0.07 & 0.00 & 0.08 & 0.94 \\
\hline $\mathrm{Ni}_{p o d}=92.323-0.558 \times \mathrm{Ni}_{\text {soil }}-9.183 \times \mathrm{pH}+2.105 \times \mathrm{OM}$ & 0.97 & 0.99 & 0.05 & 0.01 & 0.21 & 0.85 \\
\hline $\mathrm{Pb}_{\text {pod }}=1.808-0.629 \times \mathrm{Pb}_{\text {soil }}-0.023 \times \mathrm{pH}+0.261 \times \mathrm{OM}$ & 0.39 & 0.87 & 0.54 & 0.10 & 0.40 & 0.71 \\
\hline $\mathrm{Zn}_{\text {pod }}=55.093+0.050 \times \mathrm{Zn}_{\text {soil }}-5.102 \times \mathrm{pH}+1.648 \times \mathrm{OM}$ & 0.77 & 0.99 & 0.06 & 0.01 & 0.22 & 0.84 \\
\hline \multicolumn{7}{|l|}{ Leaf } \\
\hline $\mathrm{Cd}_{\text {leaf }}=0.930+0.004 \times \mathrm{Cd}_{\text {soil }}-0.093 \times \mathrm{pH}-0.002 \times \mathrm{OM}$ & 0.44 & 0.39 & 0.17 & 0.03 & 0.69 & 0.50 \\
\hline $\mathrm{Co}_{\text {leaf }}=3.561+0.048 \times \mathrm{Co}_{\text {soil }}-0.500 \times \mathrm{pH}+0.024 \times \mathrm{OM}$ & 0.87 & 0.88 & 0.10 & 0.00 & 0.10 & 0.93 \\
\hline $\mathrm{Cr}_{\text {leaf }}=16.501-0.029 \times \mathrm{Cr}_{\text {soil }}-1.356 \times \mathrm{pH}+0.095 \times \mathrm{OM}$ & 0.63 & 0.64 & 0.15 & 0.01 & 0.20 & 0.85 \\
\hline $\mathrm{Cu}_{\text {leaf }}=26.640-0.045 \times \mathrm{Cu}_{\text {soil }}-2.391 \times \mathrm{pH}-0.009 \times \mathrm{OM}$ & 0.80 & 0.77 & 0.07 & 0.01 & 0.34 & 0.74 \\
\hline $\mathrm{Fe}_{\text {leaf }}=4480.959-0.002 \times \mathrm{Fe}_{\text {soil }}-514.335 \times \mathrm{pH}-24.985 \times \mathrm{OM}$ & 0.42 & 0.52 & 0.45 & 0.04 & 0.29 & 0.77 \\
\hline $\mathrm{Mn}_{\text {leaf }}=276.061+0.050 \times \mathrm{Mn}_{\text {soil }}-19.950 \times \mathrm{pH}+5.949 \times \mathrm{OM}$ & 0.75 & 0.75 & 0.06 & 0.00 & 0.12 & 0.91 \\
\hline $\mathrm{Mo}_{\text {leaf }}=17.856+8.829 \times \mathrm{Mo}_{\text {soil }}-2.170 \times \mathrm{pH}+0.632 \times \mathrm{OM}$ & 0.90 & 0.92 & 0.07 & 0.00 & 0.00 & 1.00 \\
\hline $\mathrm{Ni}_{\text {leaf }}=24.194+0.070 \times \mathrm{Ni}_{\text {soil }}-2.771 \times \mathrm{pH}-0.078 \times \mathrm{OM}$ & 0.73 & 0.73 & 0.14 & 0.00 & 0.10 & 0.92 \\
\hline $\mathrm{Pb}_{\text {leaf }}=5.113+0.170 \times \mathrm{Pb}_{\text {soil }}-0.633 \times \mathrm{pH}-0.051 \times \mathrm{OM}$ & 0.56 & 0.50 & 0.58 & 0.04 & 0.41 & 0.68 \\
\hline $\mathrm{Zn}_{\text {leaf }}=97.118+0.009 \times \mathrm{Zn}_{\text {soil }}-9.185 \times \mathrm{pH}-0.423 \times \mathrm{OM}$ & 0.89 & 0.90 & 0.04 & 0.00 & 0.29 & 0.78 \\
\hline \multicolumn{7}{|l|}{ Stem } \\
\hline $\mathrm{Cd}_{\text {stem }}=1.770-0.037 \times \mathrm{Cd}_{\text {soil }}-0.174 \times \mathrm{pH}+0.019 \times \mathrm{OM}$ & 0.51 & 0.32 & 0.31 & 0.08 & 1.03 & 0.32 \\
\hline $\mathrm{Co}_{\text {stem }}=6.229+0.005 \times \mathrm{Co}_{\text {soil }}-0.694 \times \mathrm{pH}+0.014 \times \mathrm{OM}$ & 0.66 & 0.67 & 0.19 & 0.02 & 0.33 & 0.75 \\
\hline $\mathrm{Cr}_{\text {stem }}=16.967-0.005 \times \mathrm{Cr}_{\text {soil }}-1.877 \times \mathrm{pH}+0.110 \times \mathrm{OM}$ & 0.65 & 0.68 & 0.26 & 0.03 & 0.37 & 0.71 \\
\hline $\mathrm{Cu}_{\text {stem }}=28.016-0.029 \times \mathrm{Cu}_{\text {soil }}-2.288 \times \mathrm{pH}+0.385 \times \mathrm{OM}$ & 0.67 & 0.66 & 0.07 & 0.02 & 0.79 & 0.44 \\
\hline $\mathrm{Fe}_{\text {stem }}=2386.891-0.004 \times \mathrm{Fe}_{\text {soil }}-302.592 \times \mathrm{pH}-2.840 \times \mathrm{OM}$ & 0.56 & 0.62 & 0.46 & 0.06 & 0.50 & 0.62 \\
\hline $\mathrm{Mn}_{\text {stem }}=466.786+0.084 \times \mathrm{Mn}_{\text {soil }}-61.969 \times \mathrm{pH}+4.757 \times \mathrm{OM}$ & 0.64 & 0.62 & 0.48 & 0.02 & 0.19 & 0.86 \\
\hline $\mathrm{Mo}_{\text {stem }}=53.364+14.133 \times \mathrm{Mo}_{\text {soil }}-7.149 \times \mathrm{pH}+2.519 \times \mathrm{OM}$ & 0.87 & 0.85 & 0.16 & 0.00 & 0.02 & 0.99 \\
\hline $\mathrm{Ni}_{\text {stem }}=9.916+0.066 \times \mathrm{Ni}_{\text {soil }}-0.986 \times \mathrm{pH}+0.143 \times \mathrm{OM}$ & 0.60 & 0.59 & 0.14 & 0.04 & 0.95 & 0.35 \\
\hline $\mathrm{Pb}_{\text {stem }}=1.533+0.340 \times \mathrm{Pb}_{\text {soil }}-0.255 \times \mathrm{pH}+0.041 \times \mathrm{OM}$ & 0.46 & 0.48 & 0.29 & 0.03 & 0.41 & 0.69 \\
\hline $\mathrm{Zn}_{\text {stem }}=122.663+0.056 \times \mathrm{Zn}_{\text {soil }}-13.081 \times \mathrm{pH}+0.847 \times \mathrm{OM}$ & 0.69 & 0.69 & 0.13 & 0.01 & 0.33 & 0.75 \\
\hline \multicolumn{7}{|l|}{ Root } \\
\hline $\mathrm{Cd}_{\text {root }}=11.361-0.138 \times \mathrm{Cd}_{\text {soil }}-1.263 \times \mathrm{pH}-0.073 \times \mathrm{OM}$ & 0.61 & 0.63 & 0.20 & 0.08 & 1.06 & 0.30 \\
\hline $\mathrm{Co}_{\text {root }}=5.812+0.191 \times \mathrm{Co}_{\text {soil }}-0.434 \times \mathrm{pH}+0.616 \times \mathrm{OM}$ & 0.70 & 0.68 & 0.12 & 0.03 & 0.96 & 0.35 \\
\hline $\mathrm{Cr}_{\text {root }}=170.335-0.131 \times \mathrm{Cr}_{\text {soil }}-16.199 \times \mathrm{pH}+1.772 \times \mathrm{OM}$ & 0.61 & 0.55 & 0.20 & 0.04 & 0.69 & 0.50 \\
\hline $\mathrm{Cu}_{\text {root }}=87.997+0.915 \times \mathrm{Cu}_{\text {soil }}-11.069 \times \mathrm{pH}-0.629 \times \mathrm{OM}$ & 0.77 & 0.74 & 0.38 & 0.01 & 0.15 & 0.88 \\
\hline $\mathrm{Fe}_{\text {root }}=28452.324+0.064 \times \mathrm{Fe}_{\text {soil }}-3187.528 \times \mathrm{pH}+628.992 \times \mathrm{OM}$ & 0.61 & 0.48 & 0.20 & 0.05 & 0.68 & 0.51 \\
\hline $\mathrm{Mn}_{\text {root }}=4344.638+0.337 \times \mathrm{Mn}_{\text {soil }}-513.371 \times \mathrm{pH}-1.470 \times \mathrm{OM}$ & 0.84 & 0.84 & 0.13 & 0.00 & 0.04 & 0.97 \\
\hline $\mathrm{Mo}_{\text {root }}=16.434+1.769 \times \mathrm{Mo}_{\text {soil }}-1.980 \times \mathrm{pH}+0.725 \times \mathrm{OM}$ & 0.65 & 0.70 & 0.26 & 0.06 & 0.98 & 0.34 \\
\hline $\mathrm{Ni}_{\text {root }}=32.315+0.250 \times \mathrm{Ni}_{\text {soil }}-3.556 \times \mathrm{pH}+0.822 \times \mathrm{OM}$ & 0.82 & 0.84 & 0.09 & 0.02 & 0.88 & 0.39 \\
\hline $\mathrm{Pb}_{\text {root }}=33.307-0.452 \times \mathrm{Pb}_{\text {soil }}-3.559 \times \mathrm{pH}-0.082 \times \mathrm{OM}$ & 0.36 & 0.26 & 0.48 & 0.07 & 0.63 & 0.54 \\
\hline $\mathrm{Zn}_{\text {root }}=48.039+1.463 \times \mathrm{Zn}_{\text {soil }}-14.568 \times \mathrm{pH}+9.521 \times \mathrm{OM}$ & 0.83 & 0.83 & 0.19 & 0.00 & 0.05 & 0.96 \\
\hline
\end{tabular}

$R^{2}$ : coefficient of determination, $M E$ : model efficiency, $M N A E$ : mean normalized average error, $M N B$ : mean normalized bias

The build-up of HMs in plants is a complex process that depends on various soil properties, SS composition and application rate, species phenology and physiology, rhizosphere biochemistry, climatic factors, chelating effects and chemical speciation of 
HMs (Dolgen et al., 2007). The root system is considered the main accumulator of HMs and filter for HM uptake; it slowly accumulates HMs (particularly if present in high concentrations), thus preventing or diminishing their transport to the above-ground biomass (Eid and Shaltout, 2016; Sawidis et al., 2011). The findings of the present study confirm this observation, as almost all the estimated HMs were higher in the roots of $P$. vulgaris than in its stems, leaves and pods.

Singh et al. (2004) reported that the hyper-accumulation of HMs in a root system may be ascribed to complexation of HMs with sulfhydryl groups, resulting in less HM translocation to the shoot system. Furthermore, higher accumulation of an HM in the root of a certain plant than in its above-ground tissues may occur because the root is the first tissue to come into contact with HMs; therefore, greater accumulation occurs there (Eid and Shaltout, 2014). In a plant such as P. vulgaris, this is a preferential behaviour that prevents toxic levels of HMs from occurring in the edible shoot parts, particularly the pods, which are cooked in immature and dried stages (Huxley, 1999; Tindall, 1978).

The three HMs with the highest concentrations in the four tissues of $P$. vulgaris and the supporting soil-sludge mixture were $\mathrm{Fe}>\mathrm{Mn}>\mathrm{Zn}$, suggesting their easy uptake as a result of their higher levels in the soils and because they are essential for the growth of the studied plant (Dolgen et al., 2007; Lopes et al., 2012). In contrast, $\mathrm{Pb}$ and $\mathrm{Cd}$ tend to accumulate poorly in plant tissues and, if their uptake occurs, they accumulate in the root system (Latare et al., 2014).

The addition of SS to cultivated soil often leads to soil pollution by HMs and HM accumulation and phytotoxicity in the food supply (Yilmaz and Temizgül, 2012). The $\mathrm{HM}$ contents in the soil mixed with SS in the current work, except the $\mathrm{Cd}$ and $\mathrm{Fe}$ contents, were in the normal range reported by Allen (1989) and Kabata-Pendias (2011). In addition, most of the HM concentrations in $P$. vulgaris tissues were not in the critical ranges proposed by Kabata-Pendias (2011); however, some of them were within the normal range reported by Allen (1989) (e.g., concentrations of $\mathrm{Cu}, \mathrm{Fe}, \mathrm{Mn}, \mathrm{Pb}$ and $\mathrm{Zn}$ ). Thus, although the mixing of cultivated soil with SS may be practical for some crops, it might not be practical for others, such as $P$. vulgaris. Nevertheless, regular checking of HM levels in crop products that are cultivated in soil mixed with SS is recommended to avoid toxic effects of $\mathrm{HMs}$ in the food chain.

The BCF (root:soil ratio) is a rough estimate of HM uptake but reflects fewer details about site conditions (Zeng et al., 2011). In the current work, soil pH had a negative effect on BCF values, while soil OM had a positive effect. Significant negative $r$ coefficients between soil $\mathrm{pH}$ and BCF of HMs in the root system of $P$. vulgaris could be interpreted in the view that alkalinity decreases the availability of HMs in soil solutions while acidity increases their availability (Du Laing et al., 2008; Novotná et al., 2015; Zeng et al., 2011). In addition, the presence of significant positive $r$ coefficients between soil OM and BCF of HMs in the root system of the studied plant may be interpreted in the view that OM increases HM mobility in soil and HM uptake by plants (Singh and Agrawal, 2010). This finding is comparable to that of some previous related studies (Boshoff et al., 2014; Chaudri et al., 2007; Zeng et al., 2011). The BCF was < 1 for some metals (e.g., $\mathrm{Fe}, \mathrm{Cr}, \mathrm{Ni}, \mathrm{Cd}, \mathrm{Co}$ and $\mathrm{Cu}$ ), which means that either the accumulation capacity of the root system was low for these HMs or they were transferred in low concentrations (see Novotná et al., 2015). In contrast, the BCF was $>1$ for $\mathrm{Mn}, \mathrm{Mo}, \mathrm{Pb}$ and $\mathrm{Zn}$, which means that $P$. vulgaris is a hyper-accumulator and may be used as a phytoremediator for these metals. Regarding the TF (aerial 
tissue:root ratio), all the values were $<1$, except that for Mo, which means that the root is an effective bio-filter for these elements, while it is less effective for Mo.

Regression models are complex approaches with which we can predict the HM contents in plant tissues using some soil variables, e.g., HMs, $\mathrm{pH}$ and $\mathrm{OM}$ (Chaudri et al., 2007; Waegeneers et al., 2011). The results of the studied species indicated that the constructed models performed well for most of the estimated HM concentrations in the tissues of $P$. vulgaris, taking into account the parameters that indicate the high performance of the models ( $R^{2}, M E, M N A E$ and $p$-value). All the measured soil factors (HM, pH and $\mathrm{OM}$ ) consistently contributed to HM concentrations in the plant tissues. Similar findings were reported by other investigators (e.g., Bešter et al., 2013; Boshoff et al., 2014; dos Santos-Araujo et al., 2017; Gan et al., 2017).

To the best of the researchers knowledge, to this day, no study has been performed where regression models have been developed to forecast of HMs uptake by $P$. vulgaris from the soil amended with SS. The mathematical models for the uptake of HMs by the roots, stems, leaves and pods of $P$. vulgaris constructed here are comparable to those produced for some other crops cultivated in soil amended with SS. For example, the variability $\left(R^{2}\right)$ in $\mathrm{Cd}$ in the tissues of cucumber was $49-76 \%$ (Eid et al., 2018a), in garden pea was 25-67\% (Eid et al., 2020c), in Eruca sativa was 49-81\% (Eid et al., 2020 b), in spinach was 83-88\% (Eid et al., 2018b), compared with 44-61\% for the $P$. vulgaris tissues. In their study on the Cd models of some vegetables, Bešter et al. (2013) reported $R^{2}$ values of $41 \%$ for tomato and $90 \%$ for endive. Regarding the same element, Boshoff et al. (2014) reported an $R^{2}$ range of 10-47\% for Urtica dioica and 31-38\% for Agrostis and Poa species. In Brazil, dos Santos-Araujo et al. (2017) recorded an $R^{2}$ of $45 \%$ for lettuce and $47 \%$ for carrot. A possible reason for the lower $R^{2}$ values of some HM models in previous studies (Table 6 ) may be the large number of replicates used in model construction which results in a large amount of noise in the datasets that may influence the predictive ability of the models (Römkens et al., 2004). The pollution levels, physico-chemical characteristics of the soil at the sampling sites, soil texture, soil microbial activity, soil types with different origins and mineralogy, distinct land management practices and analytical methods used in digestion of sample materials (Du Laing et al., 2003, 2009; Kabata-Pendias, 2011) could be other reasons for the different $R^{2}$ values between HM models.

Table 6. Regression models for predicting the concentration of Cd in plants based on the concentration of $C d$ in soil and soil properties

\begin{tabular}{|c|c|c|c|c|}
\hline Plant & $n$ & Model & $R^{2}$ & Reference \\
\hline \multirow{4}{*}{ Kidney bean } & 18 & $\mathrm{Cd}_{\text {pod }}=-3.576-0.054 \times \mathrm{Cd}_{\text {soil }}+0.460 \times \mathrm{pH}+0.099 \times \mathrm{OM}(\%)$ & $54 \%$ & \multirow{4}{*}{ Present study } \\
\hline & 18 & $\mathrm{Cd}_{\text {leaf }}=0.930+0.004 \times \mathrm{Cd}_{\text {soil }}-0.093 \times \mathrm{pH}-0.002 \times \mathrm{OM}(\%)$ & $44 \%$ & \\
\hline & 18 & $\mathrm{Cd}_{\text {stem }}=1.770-0.037 \times \mathrm{Cd}_{\text {soil }}-0.174 \times \mathrm{pH}+0.019 \times \mathrm{OM}(\%)$ & $51 \%$ & \\
\hline & 18 & $\mathrm{Cd}_{\text {root }}=11.361-0.138 \times \mathrm{Cd}_{\text {soil }}-1.263 \times \mathrm{pH}-0.073 \times \mathrm{OM}(\%)$ & $61 \%$ & \\
\hline \multirow{2}{*}{$\begin{array}{l}\text { Agrostis and } \\
\text { Poa } \text { species }\end{array}$} & 37 & $\log \mathrm{Cd}_{\text {plant }}=-0.56+\left(0.58 \times \log \mathrm{Cd}_{\text {soil }}\right)$ & $38 \%$ & \multirow{2}{*}{ Boshoff et al. (2014) } \\
\hline & 37 & $\log \mathrm{Cd}_{\text {plant }}=0.08+\left(0.27 \times \log \mathrm{CaCl}_{2}[\mathrm{Cd}]_{\text {soil }}\right)$ & $31 \%$ & \\
\hline Barley & 90 & $\log \mathrm{Cd}_{\text {grain }}=0.04+0.21 \times \log \mathrm{Cd}_{\text {soil }}-0.23 \times \mathrm{pH}$ & $22 \%$ & Adams et al. (2004) \\
\hline Cabbage & 16 & $\mathrm{Cd}_{\text {plant }}=0.007+0.002 \times \mathrm{Cd}_{\text {soil }}$ & $44 \%$ & Bešter et al. (2013) \\
\hline Carrot & 54 & $\mathrm{Cd}_{\text {plant }}=0.107+0.017 \times \mathrm{Cd}_{\text {soil }}-0.00007 \times \mathrm{Mn}_{\text {soil }}$ & $47 \%$ & Bešter et al. (2013) \\
\hline \multirow{4}{*}{ Carrot } & 238 & $\mathrm{Cd}_{\text {plant }}=-0.19+0.46 \times \mathrm{Cd}_{\text {soil }}$ & $33 \%$ & \multirow{4}{*}{$\begin{array}{c}\text { dos Santos-Araujo et al. } \\
(2017)\end{array}$} \\
\hline & 238 & $\mathrm{Cd}_{\text {plant }}=0.89+0.42 \times \mathrm{Cd}_{\text {soil }}-0.17 \times \mathrm{pH}$ & $45 \%$ & \\
\hline & 238 & $\mathrm{Cd}_{\text {plant }}=0.90+0.42 \times \mathrm{Cd}_{\text {soil }}-0.17 \times \mathrm{pH}-0.01 \times \mathrm{OM}(\%)$ & $45 \%$ & \\
\hline & 238 & $\mathrm{Cd}_{\text {plant }}=0.92+0.43 \times \mathrm{Cd}_{\text {soil }}-0.18 \times \mathrm{pH}-0.01 \times \mathrm{OM}(\%)-0.04 \times$ Clay $(\%)$ & $45 \%$ & \\
\hline
\end{tabular}




\begin{tabular}{|c|c|c|c|c|}
\hline Chicory & 29 & $\mathrm{Cd}_{\text {plant }}=0.016+0.017 \times \mathrm{Cd}_{\text {soil }}$ & $60 \%$ & Bešter et al. (2013) \\
\hline \multirow{4}{*}{ Cucumber } & 18 & $\mathrm{Cd}_{\text {root }}=0.11+0.23 \times \mathrm{Cd}_{\text {soil }}-0.04 \times \mathrm{pH}+0.07 \times \mathrm{OM}(\%)$ & $49 \%$ & \multirow{4}{*}{ Eid et al. (2018a) } \\
\hline & 18 & $\mathrm{Cd}_{\text {stem }}=-0.56+0.02 \times \mathrm{Cd}_{\text {soil }}+0.07 \times \mathrm{pH}+0.03 \times \mathrm{OM}(\%)$ & $55 \%$ & \\
\hline & 18 & $\mathrm{Cd}_{\text {leaf }}=-0.14-0.002 \times \mathrm{Cd}_{\text {soil }}+0.02 \times \mathrm{pH}+0.03 \times \mathrm{OM}(\%)$ & $56 \%$ & \\
\hline & 18 & $\mathrm{Cd}_{\text {fruit }}=0.74+0.50 \times \mathrm{Cd}_{\text {soil }}-0.15 \times \mathrm{pH}+0.17 \times \mathrm{OM}(\%)$ & $76 \%$ & \\
\hline Endive & 26 & $\mathrm{Cd}_{\text {plant }}=0.089+0.032 \times \mathrm{Cd}_{\text {soil }}-0.014 \times \mathrm{OM}(\%)$ & $90 \%$ & Bešter et al. (2013) \\
\hline \multirow{2}{*}{ Eruca sativa } & 18 & $\mathrm{Cd}_{\text {leaf }}=0.326+0.204 \times \mathrm{Cd}_{\text {soil }}-0.070 \times \mathrm{pH}+0.136 \times \mathrm{OM}(\%)$ & $81 \%$ & \multirow{2}{*}{ Eid et al. (2020b) } \\
\hline & 18 & $\mathrm{Cd}_{\text {root }}=1.518-0.298 \times \mathrm{Cd}_{\text {soil }}-0.088 \times \mathrm{pH}+0.067 \times \mathrm{OM}(\%)$ & $49 \%$ & \\
\hline \multirow{3}{*}{ Garden pea } & 15 & $\mathrm{Cd}_{\text {Pod }}=4.373-0.052 \times \mathrm{Cd}_{\text {Soil }}-0.480 \times \mathrm{pH}-0.051 \times \mathrm{OM}(\%)$ & $60 \%$ & \multirow{3}{*}{ Eid et al. (2020c) } \\
\hline & 15 & $\mathrm{Cd}_{\text {Shoot }}=1.366+0.003 \times \mathrm{Cd}_{\text {Soil }}-0.133 \times \mathrm{pH}-0.020 \times \mathrm{OM}(\%)$ & $25 \%$ & \\
\hline & 15 & $\mathrm{Cd}_{\text {Root }}=-1.455+0.249 \times \mathrm{Cd}_{\text {soil }}+0.144 \times \mathrm{pH}+0.042 \times \mathrm{OM}(\%)$ & $67 \%$ & \\
\hline Hop & 13 & $\mathrm{Cd}_{\text {plant }}=0.061 \times \mathrm{Cd}_{\text {soil }}-0.28 \times \mathrm{OM}(\%)$ & $51 \%$ & Novotná et al. (2015) \\
\hline \multirow{4}{*}{ Lettuce } & 293 & $\mathrm{Cd}_{\text {plant }}=-0.06+0.39 \times \mathrm{Cd}_{\text {soil }}$ & $35 \%$ & \multirow{4}{*}{$\begin{array}{l}\text { dos Santos-Araujo et al. } \\
\text { (2017) }\end{array}$} \\
\hline & 293 & $\mathrm{Cd}_{\text {plant }}=1.10+0.44 \times \mathrm{Cd}_{\text {soil }}-0.18 \times \mathrm{pH}$ & $42 \%$ & \\
\hline & 293 & $\mathrm{Cd}_{\text {plant }}=1.35+0.48 \times \mathrm{Cd}_{\text {soil }}-0.18 \times \mathrm{pH}-0.28 \times \mathrm{OM}(\%)$ & $44 \%$ & \\
\hline & 293 & $\mathrm{Cd}_{\text {plant }}=1.11+0.39 \times \mathrm{Cd}_{\text {soil }}-0.14 \times \mathrm{pH}-0.22 \times \mathrm{OM}(\%)-0.14 \times$ Clay $(\%)$ & $47 \%$ & \\
\hline Maize & 79 & $\mathrm{Cd}_{\text {shoot }}=90.1+0.24 \times \mathrm{Cd}_{\text {Soil }}-12.9 \times \mathrm{pH}$ & $17 \%$ & $\begin{array}{l}\text { Tudoreanu and Phillips } \\
\text { (2004) }\end{array}$ \\
\hline Onion & 35 & $\mathrm{Cd}_{\text {plant }}=0.208+0.005 \times \mathrm{Cd}_{\text {soil }}-0.002 \times \mathrm{OM}(\%)-0.027 \times \mathrm{pH}$ & $85 \%$ & Bešter et al. (2013) \\
\hline Potato & 29 & $\mathrm{Cd}_{\text {plant }}=0.042+0.007 \times \mathrm{Cd}_{\text {soil }}$ & $76 \%$ & Bešter et al. (2013) \\
\hline Potato & 17 & $\begin{aligned} \mathrm{Cd}_{\text {plant }}=- & 0.018+2.46 \times \mathrm{Cd}_{\text {soil }}-0.0041 \times \text { Clay }(\%)+0.036 \times \mathrm{Zn}_{\text {soil }}+0.021 \\
& \times \mathrm{pH}: \mathrm{OM}(\%)-0.0056 \times \mathrm{Zn}_{\text {soil }}: \mathrm{pH}-0.37 \times \mathrm{Cd}_{\text {soil }} \cdot \mathrm{pH}\end{aligned}$ & $60 \%$ & Novotná et al. (2015) \\
\hline Red beet & 20 & $\mathrm{Cd}_{\text {plant }}=0.017+0.026 \times \mathrm{Cd}_{\text {soil }}$ & $67 \%$ & Bešter et al. (2013) \\
\hline Rice & 33 & $\begin{array}{c}\log \mathrm{Cd}_{\text {grain }}=0.473 \times \log \mathrm{Cd}_{\text {soil }}-0.157 \times \mathrm{pH}+0.445 \times \log \mathrm{OM}(\mathrm{g} / \mathrm{kg}) \\
-0.984\end{array}$ & $66 \%$ & Mu et al. (2020) \\
\hline Rye grass & 156 & $\mathrm{Cd}_{\text {shoot }}=35.3+0.37 \times \mathrm{Cd}_{\text {Soil }}-4.9 \times \mathrm{pH}$ & $13 \%$ & $\begin{array}{l}\text { Tudoreanu and Phillips } \\
\text { (2004) }\end{array}$ \\
\hline \multirow{2}{*}{ Spinach } & 12 & $\mathrm{Cd}_{\text {leaf }}=0.402+0.014 \times \mathrm{Cd}_{\text {soil }}-0.047 \times \mathrm{pH}+0.043 \times \mathrm{OM}(\%)$ & $88 \%$ & \multirow{2}{*}{ Eid et al. (2018b) } \\
\hline & 12 & $\mathrm{Cd}_{\text {root }}=2.144+0.060 \times \mathrm{Cd}_{\text {soil }}-0.294 \times \mathrm{pH}+0.130 \times \mathrm{OM}(\%)$ & $83 \%$ & \\
\hline Tomato & 51 & $\mathrm{Cd}_{\text {plant }}=0.020+0.002 \times \mathrm{Cd}_{\text {soil }}-0.000008 \times \mathrm{Mn}_{\text {soil }}$ & $41 \%$ & Bešter et al. (2013) \\
\hline \multirow{2}{*}{ Urtica dioica } & 66 & $\log \mathrm{Cd}_{\text {plant }}=0.26+\left(0.24 \times \log \mathrm{CaCl}_{2}[\mathrm{Cd}]_{\text {soil }}\right)$ & $10 \%$ & \multirow{2}{*}{ Boshoff et al. (2014) } \\
\hline & 68 & $\log \mathrm{Cd}_{\text {plant }}=-0.13+\left(0.69 \times \log \mathrm{Cd}_{\text {soil }}\right)-(0.87 \times \log$ clay $\%)$ & $47 \%$ & \\
\hline Wheat & 162 & $\log \mathrm{Cd}_{\text {grain }}=0.28+0.44 \times \log \mathrm{Cd}_{\text {soil }}-0.18 \times \mathrm{pH}$ & $49 \%$ & Adams et al. (2004) \\
\hline Wheat & 100 & $\log \mathrm{Cd}_{\text {plant }}=-1.75+0.59 \times \mathrm{Cd}_{\text {soil }}-0.23 \times \mathrm{OM}(\%)$ & $64 \%$ & Novotná et al. (2015) \\
\hline Wheat & 14 & $\log \mathrm{Cd}_{\text {grain }}=1.386+\log \mathrm{Cd}_{\text {soil }}-0.279 \times \mathrm{pH}$ & $85 \%$ & Liu et al. (2015) \\
\hline
\end{tabular}

\section{Conclusion}

The HM concentrations in the different tissues of $P$. vulgaris showed that most of the investigated HMs were accumulated in the plant roots rather than in the other tissues. The SS is a rich source of OM, can increase solubility of soil HMs, and therefore the use of SS as amendment must be applied carefully to avoid the accumulation of these HMs in the soil and their transfer to plants. All the TFs values were less than unity, except that for Mo, which means that the root is an effective bio-filter for these HMs, while it is less effective for Mo. Prediction models for HMs concentrations proved that $\mathrm{pH}, \mathrm{OM}$ and HMs concentrations of the soil were good predictors for the uptake of HMs by $P$. vulgaris. These models functioned well and demonstrated great levels of efficacy and low rates of error, and can be employed in examining P. vulgaris plants grown in soil amended with SS. Applying the $t$ test, there are no significant differences between the actual and predicted values of the ten HMs in P. vulgaris tissues, which reflect the best-fitting of these equations for predicting the uptake of these HMs. An 
extended field study for several growth seasons using the same SS may be required to confirm whether the repeated application of SS causes environmental problems in the future. Additionally, in future work, this study could be extended to investigate the microbial effects of SS application on soil quality and plant growth. Also, investigations of suitable methods for SS treatments are needed before the application in the agricultural system to avoid the health and environmental hazards.

Acknowledgements. This work was supported by the Deanship of Scientific Research at King Khalid University under Grant number R.G.P. 1/109/40.

\section{REFERENCES}

[1] Adams, M. L., Zhao, F. J., McGrath, S. P., Nicholson, F. A., Chambers, B. J. (2004): Predicting cadmium concentrations in wheat and barley grain using soil properties. Journal of Environmental Quality 33: 532-541.

[2] Allen, S. (1989): Chemical Analysis of Ecological Materials. - Blackwell Scientific Publications, London.

[3] Antoniadis, V., Alloway, B. (2002): The role of dissolved organic carbon in the mobility of $\mathrm{Cd}, \mathrm{Ni}$ and $\mathrm{Zn}$ in sewage sludge-amended soils. - Environmental Pollution 117: 515521.

[4] Antoniadis, V., Robinson, J., Alloway, B. (2008): Effects of short-term pH fluctuations on cadmium, nickel, lead, and zinc availability to ryegrass in a sewage sludge-amended field. - Chemosphere 71: 759-764.

[5] Aráujo, A., Monteiro, R., Carvalho, E. (2007): Effects of composted textile nodulation and nitrogen fixation of soybean and cowpea. - Bioresource Technology 98: 1028-1032.

[6] Asgari Lajayer, B., Najafi, N., Moghiseh, E., Mosaferi, M., Hadian, J. (2019): Effects of gamma irradiated and non-irradiated sewage sludge on growth characteristics, leaf chlorophyll index, and macronutrients concentrations in Basil. - Journal of Soil Science and Plant Nutrition 19: 580-591.

[7] Aydinalp, C., Marinova, S. (2003): Distribution and forms of heavy metals in some agricultural soils. - Polish Journal of Environmental Studies 12: 629-633.

[8] Belaid, N., Neel, C., Lenain, J., Buzier, R., Kallel, M., Ayoub, T., Ayadi, A., Bauduc, M. (2012): Assessment of metal accumulation in calcareous soil and forage crops subjected to long-term irrigation using treated wastewater: case of El Hajeb-Sfax, Tunisia. Agriculture, Ecosystems and Environment 158: 83-93.

[9] Bešter, P., Lobnik, F., Eržen, I., Kastelec, D., Zupan, M. (2013): Prediction of cadmium concentration in selected home-produced vegetables. - Ecotoxicology and Environmental Safety 96: 182-190.

[10] Binder, D. L., Dobermann, A., Sander, D. H., Cassman, K. G. (2002): Biosolids as nitrogen source for irrigated maize and rainfed sorghum. - Soil Science Society of America Journal 66: 531-542.

[11] Boshoff, M., De Jonge, M., Scheifler, R., Bervoets, L. (2014): Predicting As, Cd, Cu, Pb and $\mathrm{Zn}$ levels in grasses (Agrostis sp. and Poa sp.) and stinging nettle (Urtica dioica) applying soil-plant transfer models. - Science of the Total Environment 493: 862-871.

[12] Chaudri, A., McGrath, S., Gibbs, P., Chambers, B., Carlton-Smith, C., Godley, A., Bacon, J., Campbell, C., Aitken, M. (2007): Cadmium availability to wheat grain in soils treated with sewage sludge or metal salts. - Chemosphere 66: 1415-1423.

[13] Dolgen, D., Alpaslan, M., Delen, N. (2007): Agricultural recycling of treatment-plant sludge: a case study for a vegetable-processing factory. - Journal of Environmental Management 84: 274-281. 
[14] dos Santos-Araujo, S. N., Swartjes, F. A., Versluijs, K. W., Moreno, F. N., Alleoni, L. R. (2017): Soil-plant transfer models for metals to improve soil screening value guidelines valid for São Paulo, Brazil. - Environmental Monitoring and Assessment 189: 615.

[15] Du Laing, G., Tack, F. M. G., Verloo, M. G. (2003): Performance of selected destruction methods for the determination of heavy metals in reed plants (Phragmites australis). Analytica Chimica Acta 497: 191-198.

[16] Du Laing, G., De Vos, R., Vandecasteele, B., Lesage, E., Tack, F., Verloo, M. (2008): Effect of salinity on heavy metal mobility and availability in intertidal sediments of the Scheldt estuary. - Estuarine, Coastal and Shelf Science 77: 589-602.

[17] Du Laing, G., Van de Moortel, A. M. K., Moors, W., De Grauwe, P., Meers, E., Tack, F. M. G., Verloo, M. G. (2009): Factors affecting metal concentrations in reed plants (Phragmites australis) of intertidal marshes in the Scheldt estuary. - Ecological Engineering 35: 310-318.

[18] Eid, E. M., Shaltout, K. H. (2014): Monthly variations of trace elements accumulation and distribution in above- and below-ground biomass of Phragmites australis (Cav.) Trin. ex Steudel in Lake Burullus (Egypt): a biomonitoring application. - Ecological Engineering 73: 17-25.

[19] Eid, E. M., Shaltout, K. H. (2016): Bioaccumulation and translocation of heavy metals by nine native plant species grown at a sewage sludge dump site. - International Journal of Phytoremediation 18: 1075-1085.

[20] Eid, E. M., El-Bebany, A. F., Alrumman, S. A., Hesham, A., Taher, M. A., Fawy, K. F. (2017): Effects of different sewage sludge applications on heavy metal accumulation, growth and yield of spinach (Spinacia oleracea L.). - International Journal of Phytoremediation 19: 340-347.

[21] Eid, E. M., Alrumman, S. A., Farahat, E. A., El-Bebany, A. F. (2018a): Prediction models for evaluating the heavy metal uptake by cucumbers (Cucumis sativus L.) grown in agricultural soil amended with sewage sludge. - Environmental Monitoring and Assessment 190: 501.

[22] Eid, E. M., Alrumman, S. A., Galal, T. M., El-Bebany, A. F. (2018b): Prediction models for evaluating the heavy metal uptake by spinach (Spinacia oleracea L.) from soil amended with sewage sludge. - International Journal of Phytoremediation 19: 340-347.

[23] Eid, E. M., Alrumman, S. A., Galal, T. M., El-Bebany, A. F. (2019): Regression models for monitoring trace metal accumulations by Faba sativa Bernh. plants grown in soils amended with different rates of sewage sludge. - Scientific Reports 9: 5443.

[24] Eid, E. M., Khedher, K. M., Ayed, H., Arshad, M., Mouldi, A., Shaltout, K. H., Sewelam, N. A., Galal, T. M., El-Bebany, A. F., Alshehri, A. M. A. (2020a): Prediction models based on soil properties for evaluating the heavy metal uptake into Hordeum vulgare L. grown in agricultural soils amended with different rates of sewage sludge. - International Journal of Environmental Health Research. https://doi.org/10.1080/09603123.2020.1730771.

[25] Eid, E. M., Shaltout, K. H., Abdallah, S. M., Galal, T. M., El-Bebany, A. F., Sewelam, N. A. (2020b): Uptake prediction of ten heavy metals by Eruca sativa Mill. cultivated in soils amended with sewage sludge. - Bulletin of Environmental Contamination and Toxicology 104: 134-143.

[26] Eid, E. M., Shaltout, K. H., Alamri, S. A. M., Sewelam, N. A., Galal, T. M., Brima, E. I. (2020c): Prediction models for evaluating heavy metal uptake by Pisum sativum L. in soil amended with sewage sludge. - Journal of Environmental Science and Health, Part A 55: $151-160$

[27] Eid, E. M., Galal, T. M., El-Bebany, A. F. (2020d): Regression models for monitoring heavy metals accumulation by wheat (Triticum aestivum L.) plants grown in soil amended with different rates of sewage sludge. - International Journal of Phytoremediation. https://doi.org/10.1080/15226514.2020.1725866. 
[28] Farahat, E. A., Galal, T. M., Elawa, O. E., Hassan, L. M. (2017): Health risk assessment and growth characteristics of wheat and maize crops irrigated with contaminated wastewater. - Environmental Monitoring and Assessment 189: 535.

[29] Galal, T. M. (2016): Health hazards and heavy metals accumulation by summer squash (Cucurbita pepo L.) cultivated in contaminated soils. - Environmental Monitoring and Assessment 188: 434-445.

[30] Galal, T. M., Gharib, F. A., Ghazi, S. M., Mansour, K. H. (2017): Metal uptake capability of Cyperus articulatus L. and its role in mitigating heavy metals from contaminated wetlands. - Environmental Science and Pollution Research 24: 21636-21648.

[31] Gan, Y., Wang, L., Yang, G., Dai, J., Wang, R., Wang, W. (2017): Multiple factors impact the contents of heavy metals in vegetables in high natural background area of China. - Chemosphere 184: 1388-1395.

[32] Gentry, H. S. (1969): Origin of the common bean, Phaseolus vulgaris. - Economic Botany 23: 55-69.

[33] Grotto, D., Batista, B. L., Souza, J. M. O., Carneiro, M. F. H., dos Santos, D., Melo, W. J., Barbosa, Jr. F. (2015): Essential and nonessential element translocation in corn cultivated under sewage sludge application and associated health risk. - Water, Air, \& Soil Pollution 226: 261.

[34] Hoornweg, D., Bhada-Tata, P. (2012): What a Waste: A Global Review of Solid Waste Management. - Urban Development Series; Knowledge Papers No. 15. World Bank, Washington DC.

[35] Huxley, E. (1999): Dictionary of Gardening: Volume 3: L to Q. - The New Royal Horticultural Society, Macmillan Reference LTD, London.

[36] Jamali, M. K., Kazi, T. G., Arain, M. B., Afridi, H. I., Jalbani, N., Memon, A. U. R., Ansari, R., Shah, A. (2007): The feasibility of using an industrial sewage sludge produce in Pakistan as agricultural fertilizer used for cultivation of Sorghum bicolor L. - Archives of Agronomy and Soil Science 53: 659-671.

[37] Jamali, M. K., Kazi, T. G., Arain, M. B., Afridi, H. I., Jalbani, N., Kandhro, G. A., Shah, A. Q., Baig, J. A. (2009): Heavy metal accumulation in different varieties of wheat (Triticum aestivum L.) grown in soil amended with domestic sewage sludge. - Journal of Hazardous Materials 164: 1386-1391.

[38] Jones, A. L. (1999): Phaseolus Bean: Post-Harvest Operations. - FAO, Rome.

[39] Kabata-Pendias, A. (2011): Trace Elements in Soils and Plants. - CRC Press, Boca Raton.

[40] Krishnamurti, G. S., Huang, P. M., Kozak, L. M. (1999): Sorption and desorption kinetics of cadmium from soils: influence of phosphate. - Soil Science 164: 888-898.

[41] Kumar, V., Thakur, R. K., Kumar, P. (2019): Assessment of heavy metals uptake by cauliflower (Brassica oleracea var. botrytis) grown in integrated industrial effluent irrigated soils: a prediction modelling study. - Scientia Horticulturae 257: 108682.

[42] Latare, A., Kumar, O., Singh, S., Gupta, A. (2014): Direct and residual effect of sewage sludge on yield, heavy metals content and soil fertility under rice-wheat system. Ecological Engineering 69: 17-24.

[43] Legind, C. N., Trapp, S. (2010): Comparison of prediction methods for the uptake of As, $\mathrm{Cd}$ and $\mathrm{Pb}$ in carrot and lettuce. - SAR and QSAR in Environmental Research 21: 513525.

[44] Li, S., Feng, J., Tian, S., Lan, S., Fan, C., Liu, X., Xiong, Y. (2018): Tuning role and mechanism of paint sludge for characteristics of sewage sludge carbon: paint sludge as a new macro-pores forming agent. - Journal of Hazardous Materials 344: 657-668.

[45] Liu, K., Lv, J., He, W., Zhang, H., Cao, Y., Dai, Y. (2015): Major factors influencing cadmium uptake from the soil into wheat plants. - Ecotoxicology and Environmental Safety 113: 207-213. 
[46] Lopes, C., Herva, M., Franco-Uría, A., Roca, E. (2012): Multicorrelation models and uptake factors to estimate extractable metal concentrations from soil and metal in plants in pasture lands fertilized with manure. - Environmental Pollution 166: 17-22.

[47] Mamais, D., Kouzeli-Katsiri, A., Christoulas, D. G., Andreadakis, A. D., Aftias, E. (2000): Evaluation of agricultural utilization of the sludge produced at Psyttalia wastewater treatment plant. - Water Science and Technology 42: 21-28.

[48] Mu, T., Zhou, T., Li, Z., Hu, P., Luo, Y., Christie, P., Wu, L. (2020): Prediction models for rice cadmium accumulation in Chinese paddy fields and the implications in deducing soil thresholds based on food safety standards. - Environmental Pollution 258: 113879.

[49] Novotná, M., Mikeš, O., Komprdová, K. (2015): Development and comparison of regression models for the uptake of metals into various field crops. - Environmental Pollution 207: 357-364.

[50] Rafiq, M. T., Aziz, R., Yang, Z., Xiao, W., Rafiq, M. K., Ali, B., Li, T. (2014): Cadmium phytoavailablity to rice (Oryza sativa L.) grown in representative Chinese soils. A model to improve soil environmental quality guidelines for food safety. - Ecotoxicology and Environmental Safety 103: 101-107.

[51] Ramadan, M. A. E., Al-Ashkar, E. A. (2007): The effect of different fertilizers on the heavy metals in soil and tomato plant. - Australian Journal of Basic and Applied Sciences 1: 300-306.

[52] Rehman, R. A., Rizwan, M., Qayyum, M. F., Ali, S., Zia-ur-Rehman, M., Zafar-ul-Hye, M., Hafeez, F., Iqbal, M. F. (2018): Efficiency of various sewage sludges and their biochars in improving selected soil properties and growth of wheat (Triticum aestivum). Journal of Environmental Management 223: 607-613.

[53] Römkens, P. F. A. M., Groenenberg, J. E., Bril, J., Vries, W. (2004): Derivation of partition equations to calculate heavy metal speciation and solubility in soils. - Alterra Report 305,Wageningen.

[54] Sawidis, T., Breuste, J., Mitrovic, M., Pavlovic, P., Tsigaridas, K. (2011): Trees as bioindicator of heavy metal pollution in three European cities. - Environmental Pollution 159: 3560-3570.

[55] Sharma, B., Sarkar, A., Singh, P., Singh, R. P. (2017): Agricultural utilization of biosolids: a review on potential effects on soil and plant grown. - Waste Management 64: 117-132.

[56] Singh, R. P., Agrawal, M. (2007): Effects of sewage sludge amendment on heavy metal accumulation and consequent responses of Beta vulgaris plants. - Chemosphere 67: 2229-2240.

[57] Singh, R. P., Agrawal, M. (2008): Potential benefits and risks of land application of sewage sludge. - Waste Management 28: 347-358.

[58] Singh, R. P., Agrawal, M. (2010): Effect of different sewage sludge applications on growth and yield of Vigna radiata L. field crop: metal uptake by plant. - Ecological Engineering 36: 969-972.

[59] Singh, S., Saxena, R., Pandey, K., Bhatt, K., Sinha, S. (2004): Response of antioxidants in sunflower (Helianthus annuus L.) grown on different amendments of tannery sludge: its metal accumulation potential. - Chemosphere 57: 1663-1673.

[60] SPSS (2006): SPSS Base 15.0 User's Guide. - SPSS Inc., Chicago.

[61] Tindall, H. D. (1978): Commercial Vegetable Growing. - The English Language Book Society and Oxford University Press, Oxford.

[62] Tudoreanu, L., Phillips, C. J. C. (2004): Empirical models of cadmium accumulation in maize, rye grass and soya bean plants. - Journal of the Science of Food and Agriculture 84: 845-852.

[63] Usman, A., Kuzyakov, Y., Stahr, K. (2008): Sorption, Desorption, and Immobilization of Heavy Metals by Artificial Soil. - University of Hohenhiem, Stuttgart. 
[64] Waegeneers, N., Ruttens, A., De Temmerman, L. (2011): A dynamic model to calculate cadmium concentrations in bovine tissues from basic soil characteristics. - Science of the Total Environment 409: 2815-2823.

[65] Wang, X., Shan, X., Zhang, S., Wen, B. (2004): A model for evaluation of the phytoavailability of trace elements to vegetables under the field conditions. Chemosphere 55: 811-822.

[66] Wilke, B. M. (2005): Determination of Chemical and Physical Soil Properties. - In: Margesin, R., Schinner, F. (eds.) Manual for Soil Analysis - Monitoring and Assessing Soil Bioremediation. Springer-Verlag, Heidelberg, pp. 47-95.

[67] Yang, H., Li, Z., Lu, L., Long, J., Liang, Y. (2013): Cross-species extrapolation of prediction models for cadmium transfer from soil to corn grain. - PLoS ONE 8: e80855.

[68] Yilmaz, D. D., Temizgül, A. (2012): Effects of municipal sewage sludge doses on the chlorophyll contents and heavy metal concentration of sugar beet (Beta vulgaris var. saccharifera). - Bioremediation Journal 16: 131-140.

[69] Zeng, F., Ali, S., Zhang, H., Ouyang, Y., Qiu, B., Wu, F., Zhang, G. (2011): The influence of $\mathrm{pH}$ and organic matter content in paddy soil on heavy metal availability and their uptake by rice plants. - Environmental Pollution 159: 84-91.

[70] Zhang, S., Song, J., Gao, H., Zhang, Q., Lv, M. C., Wang, S., Liu, G. (2016): Improving prediction of metal uptake by Chinese cabbage (Brassica pekinensis L.) based on a soilplant stepwise analysis. - Science of the Total Environment 569-570: 1595-1605.

[71] Zhao, K. L., Liu, X. M., Xu, J. M., Selim, H. M. (2010): Heavy metal contaminations in a soil-rice system: identification of spatial dependence in relation to soil properties of paddy fields. - Journal of Hazardous Materials 181: 778-787.

\section{APPENDIX}

Appendix 1. Selected chemical properties of sewage sludge and agricultural soil used in the pot experiment (means \pm standard error, $n=3$ )

\begin{tabular}{c|c|c}
\hline Properties & Agricultural soil & Sewage sludge \\
\hline $\mathrm{pH}$ & $8.7 \pm 0.02$ & $7.0 \pm 0.02$ \\
Organic matter $(\%)$ & $0.9 \pm 0.2$ & $65.0 \pm 0.9$ \\
$\mathrm{Cd}\left(\mathrm{mg} \mathrm{kg}^{-1}\right)$ & $2.9 \pm 0.1$ & $1.2 \pm 0.1$ \\
$\mathrm{Co}\left(\mathrm{mg} \mathrm{kg}^{-1}\right)$ & $35.5 \pm 1.1$ & $25.9 \pm 1.3$ \\
$\mathrm{Cr}\left(\mathrm{mg} \mathrm{kg}^{-1}\right)$ & $134.3 \pm 0.7$ & $176.2 \pm 1.9$ \\
$\mathrm{Cu}\left(\mathrm{mg} \mathrm{kg}^{-1}\right)$ & $15.0 \pm 0.6$ & $162.6 \pm 2.3$ \\
$\mathrm{Fe}\left(\mathrm{mg} \mathrm{g}^{-1}\right)$ & $42.4 \pm 0.5$ & $24.1 \pm 0.5$ \\
$\mathrm{Mn}\left(\mathrm{mg} \mathrm{kg}^{-1}\right)$ & $677.3 \pm 3.2$ & $560.7 \pm 9.8$ \\
$\mathrm{Mo}\left(\mathrm{mg} \mathrm{kg}^{-1}\right)$ & $1.1 \pm 0.0$ & $0.9 \pm 0.0$ \\
$\mathrm{Ni}\left(\mathrm{mg} \mathrm{kg}^{-1}\right)$ & $68.1 \pm 3.7$ & $138.7 \pm 3.7$ \\
$\mathrm{~Pb}\left(\mathrm{mg} \mathrm{kg}^{-1}\right)$ & $3.5 \pm 0.4$ & $671.1 \pm 6.2$ \\
$\mathrm{Zn}\left(\mathrm{mg} \mathrm{kg}^{-1}\right)$ & $77.2 \pm 1.9$ & $667.6 \pm 13.4$ \\
\hline
\end{tabular}

